\title{
SPECTRAL SECTIONS AND HIGHER ATIYAH-PATODI-SINGER INDEX THEORY ON GALOIS COVERINGS
}

\author{
E. Leichtnam and P. Piazza
}

\begin{abstract}
In this paper we consider $\Gamma \rightarrow \widetilde{M} \rightarrow M$, a Galois covering with boundary and $\widetilde{\not D}$, a $\Gamma$-invariant generalized Dirac operator on $\widetilde{M}$. We assume that the group $\Gamma$ is of polynomial growth with respect to a word metric. By employing the notion of noncommutative spectral section associated to the boundary operator $\widetilde{D}_{0}$ and the $b$-calculus on Galois coverings with boundary, we develop a higher Atiyah-PatodiSinger index theory. Our main theorem extends to such $\Gamma$-Galois coverings with boundary the higher index theorem of Connes-Moscovici.
\end{abstract}

\section{Contents.}

0 . Introduction.

1. Geometric preliminaries.

2. Noncommutative spectral sections and $\mathcal{B}^{\infty}$-pseudodifferential calculus.

3. $\mathcal{P}$-higher eta invariants.

4. Proof of the technical Lemmas.

5. Variation properties of $\mathcal{P}$-higher eta invariants and the noncommutative spectral flow.

6. Higher Atiyah-Patodi-Singer index classes.

7. $b$-Chern character, transgression formulae and the higher APS index theorem.

\section{Introduction}

The goal of this paper is to state and prove a general higher Atiyah-PatodiSinger ( $\equiv$ APS) index theorem on Galois coverings with boundary. We begin by recalling the context and the historical development of this problem.

Let $\Gamma \rightarrow \widetilde{N} \rightarrow N$ be a Galois covering, with $N$ a closed compact manifold. As explained for example in [Co], the Connes-Moscovici higher index theorem on $\widetilde{N}$ [CoMo] can be viewed as a noncommutative family index theorem, the base being the noncommutative space defined by the 
reduced $C^{*}$-algebra of $\Gamma: C_{r}^{*}(\Gamma)$. In particular when the covering group $\Gamma$ is commutative $\left(=\mathbb{Z}^{k}\right)$ the higher index theorem reduces to a family index theorem with parameter space equal to the dual group $\widehat{\Gamma}$ (a torus $T^{k}$ ). This is the case originally treated by Lustzig $[\mathrm{Lu}]$ in order to apply higher index theory to the question of the homotopy invariance of Novikov's higher signatures.

Let us pass to manifolds with boundary. The first result in APS family (i.e. commutative) index theory is due to Bismut and Cheeger. Let $\not D=\left(D_{z}\right)_{z \in B}$ be a family of Dirac-type operators on even dimensional manifolds with boundary. The operators $D_{z}$ are odd with respect to the $\mathbb{Z}_{2}$-grading of the relevant Clifford modules. Let $\not_{0}=\left(D_{0, z}\right)_{z \in B}$ be the boundary family. Building on Cheeger's analysis of the heat kernel on manifolds with conical singularities $[\mathrm{C}]$ and Bismut's superconnection heat kernel proof of the family index theorem [Bi] and assuming moreover that the boundary family $\not_{0}$ is invertible, Bismut and Cheeger [BiC1,2] define an index bundle associated to $\not D^{+}$and compute its Chern character. In [BiC3] an extension of their result is stated assuming only that the null spaces of the boundary family are of constant rank (see [MPi2] for a full proof of the latter result). A noncommutative version of Bismut-Cheeger family index theorem is presented in [LPi1]. Let $\Gamma \rightarrow \widetilde{M} \rightarrow M$ be an even dimensional Galois covering with boundary $\partial \widetilde{M} \rightarrow \partial M$. Assume the covering group $\Gamma$ to be of polynomial growth. This means that for a word metric \|\| on $\Gamma$, the cardinality of the ball $B(e, R)$ is of polynomial growth with respect to $R$. Gromov has proved [Gr] that this condition is equivalent to the property that $\Gamma$ is virtually nilpotent: i.e. contains a nilpotent subgroup of finite index. We consider the following subalgebra of the reduced $C^{*}$-algebra $C_{r}^{*}(\Gamma)$ :

$$
\mathcal{B}^{\infty}=\left\{f: \Gamma \rightarrow \mathbb{C} / \forall L \in N, \sup _{\gamma \in \Gamma}(1+\|\gamma\|)^{L}|f(\gamma)|<+\infty\right\} .
$$

It is well known that $\mathcal{B}^{\infty}$ is closed under holomorphic functional calculus. Roughly speaking, $\mathcal{B}^{\infty}$ is the set of $C^{\infty}$-functions on the noncommutative space $C_{r}^{*}(\Gamma)$. Let $\not D$ be a generalized Dirac-type operator acting on a Clifford module $E$ on $M$, both having a product structure near $\partial M$, and let $\not_{0}$ be the associated boundary operator. We will use the following two infinite dimensional bundles

$$
\mathcal{E}=E \otimes_{\mathbb{C}}\left(\widetilde{M} \times_{\Gamma} C_{r}^{*}(\Gamma)\right) \rightarrow M,\left.\quad \mathcal{E}_{0} \equiv \mathcal{E}\right|_{\partial M} \rightarrow \partial M,
$$

whose fiber over a point $z \in M$ is $E_{z} \otimes C_{r}^{*}(\Gamma)$. We can also consider the bundles $\mathcal{E}^{\infty}, \mathcal{E}_{0}^{\infty}$ defined in terms of $\mathcal{B}^{\infty}$ instead of $C_{r}^{*}(\Gamma)$. To the two Dirac 
operators $\not D, \not D_{0}$ we associate, first on $M$ two $C_{r}^{*}(\Gamma)$-linear operators $\not{D}, \not_{0}$ acting respectively on the sections of $\mathcal{E}$ and $\mathcal{E}_{0}$, second on the covering $\widetilde{M}$, two (lifted) $\Gamma$-invariant operators $\widetilde{D}, \widetilde{D}_{0}$. We now recall the main result of [LPi1].

Theorem 0.1. Let $\widetilde{D}, \not{D}, \widetilde{D}_{0}, \mathcal{D}_{0}$ be Dirac operators as above. We assume that there exists a $\delta>0$ such that

$$
\left.\operatorname{spec}_{L^{2}}\left(\widetilde{D}_{0}\right) \cap\right]-\delta, \delta[=\{0\} .
$$

Then

(1) There is a well defined index class $\operatorname{Ind}\left(\not{D}^{+}\right) \in \mathcal{K}_{0}\left(\mathcal{B}^{\infty}\right) \cong \mathcal{K}_{0}\left(C_{r}^{*}(\Gamma)\right)$.

(2) The null space of $\not{D}_{0}$ acting on $C^{\infty}\left(\partial M, \mathcal{E}_{0}^{\infty}\right)$ is a projective, finitely generated $\mathcal{B}^{\infty}$-module.

(3) For the Chern character of $\operatorname{Ind}\left(\not{D}^{+}\right)$, in the noncommutative topological de Rham homology of $\mathcal{B}^{\infty}$, the following formula holds

$$
\operatorname{Ch}\left(\operatorname{Ind}\left(\not{D}^{+}\right)\right)=\int_{M} \widehat{A} \wedge \mathrm{Ch}^{\prime}(E) \wedge \omega-\frac{1}{2}\left(\tilde{\eta}+\operatorname{Ch}\left(\operatorname{null}\left(\mathcal{D}_{0}\right)\right)\right) \text { in } \bar{H}_{*}\left(\mathcal{B}^{\infty}\right) \text {. }
$$

For the definition of $\bar{H}_{*}\left(\mathcal{B}^{\infty}\right)$ see $[\mathrm{K}]$. In this index formula the higher eta invariant $\tilde{\eta}$ of Lott [Lo2] appears. The biform $\omega$, also introduced by Lott in his superconnection heat-kernel proof of the Connes-Moscovici [CoMo] higher index theorem [Lo1], can be explicitly described. Theorem 0.1 settles (and also extends from the invertible to the gap case) a conjecture by Lott [Lo2]. The proof of Theorem 0.1 rests ultimately on Lott's noncommutative superconnection formalism, on an extension of Melrose's $b$-calculus to Galois coverings, and on a $\mathcal{B}^{\infty}$-b-calculus on the compact manifold $M$. Much of [LPi1] is in fact devoted to a rigorous development of these analytic techniques.

The goal of this paper is to extend Theorem 0.1 to the general case by removing completely the gap assumption (0.2). It is important to observe that this latter assumption (0.2) is the analogue, in the noncommutative setting, of the Bismut-Cheeger hypothesis that the null spaces of the boundary family have constant rank. Now, in the family case treated by Bismut and Cheeger this constant rank assumption was necessary in order to define a smooth family of APS boundary value problems. In order to develop a general APS family index theory, i.e. with no assumptions on the boundary family, it is necessary to consider generalized APS boundary conditions. The key concept in this direction, that of spectral section, was introduced in $[\mathrm{MPi} 2,3]$. We now recall the definition. 
Let $D=\left(D_{z}\right)_{z \in B}$ be a smooth family of formally self-adjoint elliptic differential operators parametrized by a compact space $B$. Each operator $D_{z}$ acts on the sections of a hermitian vector bundle $F_{z}$ over a closed riemannian manifold $N_{z}$. The boundary family $\not_{0}$ associated to a family $\not D$ of Dirac operators on even dimensional manifolds with boundary is a fundamental and motivating example of such a $D$. A spectral section $P$ for $D$ is a smooth family $P=\left(P_{z}\right)_{z \in B}$ of self-adjoint projections with $P_{z} \in \Psi^{0}\left(N_{z} ; F_{z}\right)$ and satisfying the following property:

$$
\exists R \in \mathbb{R} \text { such that } D_{z} u=\lambda u \Rightarrow \begin{cases}P u=u & \text { if } \lambda>R \\ P u=0 & \text { if } \lambda<-R .\end{cases}
$$

This means that each operator $P_{z}$ is equal to the identity on the eigenfunctions of $D_{z}$ corresponding to large positive eigenvalues and equal to 0 on those corresponding to large negative eigenvalues. Each $P_{z}$ is a finite rank perturbation of the spectral projection $\Pi_{\geq, z}$ corresponding to the non-negative eigenvalues of $D_{z}$.

The family $D$ is a family of self-adjoint Fredholm operators parametrized by $B$; it therefore defines an index class $\operatorname{Ind}(D) \in K^{1}(B)$. One of the main results in [MPi2] asserts that a spectral section for $D$ exists if and only if $\operatorname{Ind}(D)=0$ in $K^{1}(B)$. It is not difficult to see that if $\operatorname{Ind}(D)=0$ then the set of spectral sections $\operatorname{SP}(D)$ associated to $D$ is infinite. For a boundary Dirac family such as $\not_{0}$ it is always the case that $\operatorname{Ind}\left(\not_{0}\right)=0$; thus this family admits an infinite number of spectral sections. Once a spectral section $P$ for $\not_{0}$ has been fixed, it is possible to define a smooth family of generalized APS boundary value problems and an index class $\operatorname{Ind}\left(\not D^{+}, P\right) \in K^{0}(B)$. The $b$-calculus is then heavily used in [MPi2] to give a formula for the Chern character of this index class (see [MPi3] for the odd dimensional case).

The concept of spectral section associated to a family of self-adjoint elliptic operators has also been used by Dai and Zhang in order to define the higher spectral flow of a curve of families; the higher spectral flow is an element of $K^{0}(B)=K_{0}\left(C^{0}(B)\right)$ [DZ]. We briefly recall their definition. Let $\left\{\not D_{u}\right\}, u \in[0,1]$ be a curve of families of self-adjoint Dirac operators, $\not D_{u}=\left(\left(D_{u}\right)_{z}\right)_{z \in B}$. Assume that $\operatorname{Ind}\left(\not D_{0}\right)=0$ in $K^{1}(B)$. Thus $\operatorname{Ind}\left(D_{u}\right)=0$ $\forall u$. For the total family defined by $\left\{\not_{u}\right\}$ on $[0,1] \times B$ we also have the vanishing of the $K^{1}([0,1] \times B)$-index. Thus there exists a total spectral section $P=\left\{P_{u}\right\}, P_{u}$ being a spectral section of the family $\not_{u}$ for each $u \in[0,1]$. Let $Q_{0}, Q_{1}$ be spectral sections of $\not_{0}, \not_{1}$ respectively. The higher 
spectral flow $([\mathrm{DZ}])$ between the pairs $\left(\not D_{0}, Q_{0}\right),\left(\not D_{1}, Q_{1}\right)$ is by definition

$$
\operatorname{sf}\left(\left\{D_{u}\right\} ; Q_{0}, Q_{1}\right)=\left[Q_{1}-P_{1}\right]-\left[Q_{0}-P_{0}\right] \in K^{0}(B) .
$$

This class depends on $\left\{D_{u}\right\}, Q_{0}, Q_{1}$ (of course) but not on the choice of $P=\left\{P_{u}\right\}$. When $B$ is reduced to a point the higher spectral flow becomes an integer $\left(\in K^{0}(\{\mathrm{pt}\})\right)$ if $Q_{j}, j=0,1$ is the spectral projection onto the non-negative eigenfunctions of $\not_{j}$, then this integer is equal to the net number of eigenvalues that change sign when $u$ varies from 0 to 1 (this is the spectral flow as defined in [APS3]).

Motivated by [DZ] Wu has extended the notion of spectral section from $C^{0}(B)$-Hilbert modules, as above, to arbitrary $A$-Hilbert modules, with $A$ a unital $C^{*}$-algebra [W1]. Extending to this context the results in [MPi2], Wu has then defined a noncommutative higher spectral flow in $K_{0}(A)$, generalizing the work of [DZ]. The notion of noncommutative spectral section, as defined by $\mathrm{Wu}$, will be the starting point of this article, the contents of which shall now be described.

We adopt the notation introduced before Theorem 0.1. As already remarked the boundary operator $\widetilde{D}_{0}$ defines in a natural way a $C^{*}(\Gamma)$ linear operator $\mathcal{D}_{0}: C^{\infty}\left(\partial M, \mathcal{E}_{0}\right) \rightarrow C^{\infty}\left(\partial M, \mathcal{E}_{0}\right)$. It can be extended to $L^{2}\left(\partial M, \mathcal{E}_{0}\right)$ thus defining an unbounded self-adjoint regular operator on the Hilbert $C_{r}^{*}(\Gamma)$-module $L^{2}\left(\partial M, \mathcal{E}_{0}\right)$. This is one example of the general noncommutative framework considered in [W1]. In sect. 2, we begin by proving that $\operatorname{Ind}\left(\mathcal{D}_{0}\right)=0 \in K_{1}\left(C_{r}^{*}(\Gamma)\right)$, using the fact that $\mathcal{D}_{0}$ is a boundary operator. Thus, according to the noncommutative analogue of the result presented above, it admits a noncommutative spectral section $\mathcal{P}$ as defined in [W1]. The next task is to show that such a spectral section can be chosen in the $\mathcal{B}^{\infty}$-calculus. This is a technical but important point; it is achieved (always in sect. 2) by constructing a $\mathcal{B}^{\infty}$-smoothing self-adjoint operator $\mathcal{A}^{0}$, on $\partial M$, such that the projection onto the positive part of the invertible operator $\mathcal{D}_{0}+\mathcal{A}^{0}$ is a spectral section $\mathcal{P}$ belonging to the $\mathcal{B}^{\infty}$ pseudodifferential calculus. In sect. 3 and sect. 4 , we define the $\mathcal{P}$-higher eta invariant $\widehat{\eta}_{\mathcal{P}}$ as a time integral involving $\not_{0}+\mathcal{A}^{0}$ and Lott's superconnection. Much of these two sections is devoted to the proof of the convergence of the time integral. In sect. 5 , we first investigate the dependence of the $\mathcal{P}$-higher eta invariant on the choice of the spectral section $\mathcal{P}$ (Theorem 5.1): if $\mathcal{Q}$ is another $\mathcal{B}^{\infty}$-spectral section the formal difference $[\mathcal{P}-\mathcal{Q}]$ defines a class in $K_{0}\left(\mathcal{B}^{\infty}\right)$ and we have

$$
\widehat{\eta}_{\mathcal{P}}-\widehat{\eta}_{\mathcal{Q}}=2 \operatorname{Ch}[\mathcal{P}-\mathcal{Q}] \in \bar{H}_{*}\left(\mathcal{B}^{\infty}\right) .
$$

In the spirit of [DZ], with notation parallel to those of formula (0.5), we 
also establish the following formula for the Chern character of the noncommutative spectral flow of [W1]:

$2 \operatorname{Ch}\left(\operatorname{sf}\left(\left\{\not{D}_{u}\right\} ; Q_{0}, Q_{1}\right)\right)=\widehat{\eta}_{\mathcal{Q}_{1}}\left(\mathcal{D}_{1}\right)-\widehat{\eta}_{\mathcal{Q}_{0}}\left(\mathcal{D}_{0}\right)-\int_{0}^{1} a_{0}(u) d u$ in $\bar{H}_{*}\left(\mathcal{B}^{\infty}\right)$ where $a_{0}(u)$ is a "local term" belonging to the space of noncommutative differential forms of $\mathcal{B}^{\infty}$.

In sect. 6 , we associate (in a natural way) to $\mathcal{A}^{0}$ an operator $\mathcal{A}_{\mathcal{P}}^{+}$on $M$ such that $\not{D}^{+}+\mathcal{A}_{\mathcal{P}}^{+}$is $C_{r}^{*}(\Gamma)$-Fredholm $([\mathrm{MiF}])$ and admits $\not{D}_{0}+\mathcal{A}^{0}$ as boundary operator. By using the $b$-calculus and by adapting results of $[\mathrm{MiF}]$ to the $\mathcal{B}^{\infty}$-calculus we show more precisely that $\not{D}^{+}+\mathcal{A}_{\mathcal{P}}^{+}$defines an index class $\operatorname{Ind}\left(\mathcal{D}^{+}, \mathcal{P}\right)$ which depends only on $\mathcal{P}$ and belongs to $K_{0}\left(\mathcal{B}^{\infty}\right)$. This means that $\operatorname{Ch}\left(\operatorname{Ind}\left(\not{D}^{+}, \mathcal{P}\right)\right)$ is well defined in $\bar{H}_{*}\left(\mathcal{B}^{\infty}\right)$. In sect. 7 , we state and prove our main result, Theorem 7.6, which gives an AtiyahPatodi-Singer formula for this homology class,

$$
\operatorname{Ch}\left(\operatorname{Ind}\left(\not{D}^{+}, \mathcal{P}\right)\right)=\int_{M} \widehat{A} \wedge \mathrm{Ch}^{\prime}(E) \wedge \omega-\frac{1}{2} \widehat{\eta}_{\mathcal{P}} \quad \text { in } \quad \bar{H}_{*}\left(\mathcal{B}^{\infty}\right) .
$$

As a corollary of (0.7) and (0.6) we also obtain,

$$
\operatorname{Ch}\left(\operatorname{Ind}\left(\not{D}^{+}, \mathcal{P}\right)\right)-\operatorname{Ch}\left(\operatorname{Ind}\left(\not{D}^{+}, \mathcal{Q}\right)\right)=\operatorname{Ch}[\mathcal{Q}-\mathcal{P}]
$$

We end the paper by showing how Theorem 0.1 , and formula (0.3) in particular, can be deduced from (0.7).

The results of this paper were announced in [LPi2].

REMARK. Although the proofs presented in the present paper do depend on a few technical analytic points fully developed in [LPi1], the statements and in fact the whole structure can be followed independently of [LPi1].

Acknowledgements. It is a pleasure to thank Jean-Michel Bismut and John Lott for interesting discussions, Richard Melrose for helpful comments on the manuscript and Fangbing Wu for explaining to us part of his work [W1]. Much of this work was done during a number of visits of the first author to the Università "La Sapienza" and of the second author to École Normale Supérieure. Financial support for these visits was provided by a CNR-CNRS cooperation project and by École Normale Supérieure. We take this opportunity to thank these institutions.

\section{Geometric Preliminaries}

We consider a compact closed riemannian manifold $N$ and $\pi: \widetilde{N} \rightarrow N$ a Galois $\Gamma$-covering. For the time being we only assume the discrete group 
$\Gamma$ to be finitely presented. The dimension of $N$ may be either even or odd. Let $E$ be a hermitian Clifford module over $N$ endowed with a unitary Clifford connection. Let $\not D$ be the associated Dirac operator. If $N$ is evendimensional then both $E$ and $\not D$ are assumed to be $\mathbb{Z}_{2}$-graded. We denote by $\Upsilon$ the grading operator; thus $\Upsilon^{2}=$ Id and $E^{ \pm}=\operatorname{ker}(\Upsilon \pm$ Id). If $N$ is odd dimensional then we consider the bundle of $\mathbb{C}$-vector spaces $E_{\sigma}=$ $E \otimes \mathrm{Cl}(1)$. Here $\mathrm{Cl}(1)$ is the complex Clifford algebra of $\mathbb{C}$, it is generated, as a complex vector space, by 1 and $\sigma$ with $\sigma^{2}=1$. There is a natural bundle isomorphism $E_{\sigma} \rightarrow E \oplus E$ under which $\sigma$ becomes the matrix

$$
\sigma=\left(\begin{array}{ll}
0 & 1 \\
1 & 0
\end{array}\right)
$$

The bundle $E_{\sigma}$ has an obvious $\mathbb{Z}_{2}$-grading.

We denote by $\widetilde{D}$ the lifted $\Gamma$-invariant Dirac operator on the covering $\widetilde{N}$ acting on the sections of the lifted bundle $\widetilde{E}=\pi^{*}(E) \rightarrow \widetilde{N}$. More generally we consider $\Gamma$-invariant pseudodifferential operators acting between the sections of two $\Gamma$-invariant vector bundles $\widetilde{F}_{0}, \widetilde{F}_{1}$ over $\widetilde{N}$ (lifts to $\widetilde{N}$ of vector bundles $F_{0}, F_{1}$ over $\left.N\right)$. We denote by $\Psi_{\Gamma}^{*}\left(\widetilde{N} ; \widetilde{F}_{0}, \widetilde{F}_{1}\right)$ the space of all such operators.

Let $\Lambda$ be the reduced $C^{*}$-algebra of the group $\Lambda \equiv C_{r}^{*}(\Gamma)$. To each vector bundle $F$ over $N$, we will associate the bundle of projective finitely generated $\Lambda$-modules $\mathcal{F} \rightarrow N$ with fiber $\mathcal{F}_{z}=F_{z} \otimes\left(C_{r}^{*}(\Gamma) \times_{\Gamma} \widetilde{N}\right)_{z}$, with $z \in N$. We denote by $\Psi_{\Lambda}^{*}\left(N ; \mathcal{F}_{0}, \mathcal{F}_{1}\right)$ the space of classical $\Lambda$-pseudodifferential operators defined by Mishenko-Fomenko [MiF]. Our Dirac operator $\not D$ in $\operatorname{Diff}^{1}(N, E)$ induces (in an obvious way) a $\Lambda$-differential operator $\not{D}$ in $\operatorname{Diff}_{\Lambda}^{1}(N, \mathcal{E})$.

In the second part of the paper, beginning with sect. 4 , we shall consider a smooth connected compact manifold with boundary $M$ and $\Gamma \rightarrow \widetilde{M} \rightarrow M$ a Galois covering of $M$ with covering group equal to $\Gamma$. We fix a boundary defining function $\tilde{x} \in C^{\infty}(\widetilde{M})$ by lifting from $M$ a boundary defining function $x \in C^{\infty}(M)$. Let $g_{M} \in \Gamma^{\infty}\left(M,{ }^{b} T^{*} M \otimes{ }^{b} T^{*} M\right)$ be an exact $b$-metric on $M$. Thus (we can assume that)

$$
g_{M}=(d x / x)^{2}+h_{M}
$$

with $h_{M} \in C^{\infty}\left(M, T^{*} M \otimes T^{*} M\right)$. The lift of $g_{M}$ to $\widetilde{M}$ is an exact $b$-metric which is furthermore $\Gamma$-invariant. We denote this lifted metric by $g$. As in the closed case we consider on $M$ a unitary Clifford module $E$ endowed with a unitary connection $\nabla^{E}$ which is Clifford with respect to the $b$-LeviCivita connection associated to the $b$-metric $g_{M}$. We assume that $\nabla_{x \partial_{x}}^{E} \equiv 0$ 
on $\partial M$. We denote by $\not D$ the generalized Dirac operator associated to these data. The lift of $\not D$ to the covering $\widetilde{M}$ is a $\Gamma$-invariant $b$-differential operator $\widetilde{D}$; it is precisely the $b$-Dirac operator associated to the lifted data on $\widetilde{E} \equiv \pi^{*}(E)$. The grading in the odd and even dimensional case is as in the closed case treated above.

Suppose now that $M$ is even dimensional. The Clifford bundle associated to $T^{*}(\partial \widetilde{M})$ acts in a natural way on $\widetilde{E}_{\mid \partial \widetilde{M}}$ :

$$
\forall e \in \widetilde{E}_{\mid \partial \widetilde{M}}, \quad \forall \eta \in T^{*} \partial \widetilde{M}, \operatorname{cl}_{\partial}(\eta)\left(e_{\mid \partial \widetilde{M}}\right) \equiv \operatorname{cl}\left(i \frac{d \tilde{x}}{\tilde{x}}\right) \operatorname{cl}(\eta)\left(e_{\mid \partial \widetilde{M}}\right) .
$$

We define $\widetilde{E}_{0}$ to be $\widetilde{E}_{\mid \partial \widetilde{M}}^{+}$. It is a unitary Clifford bundle with respect to $\operatorname{cl}_{\partial}(\cdot)$. It is endowed with the induced Clifford connection. We denote by $\widetilde{D}_{0}$ the associated $\Gamma$-invariant Dirac operator, we call it the boundary operator of $\widetilde{D}$. Finally, we identify $\widetilde{E}_{\mid \partial \widetilde{M}}^{-}$with $\widetilde{E}_{0}$ through Clifford multiplication by $\operatorname{cl}(i d \tilde{x} / \tilde{x})$. With these identifications, that will always be used in the rest of the paper, the indicial family $I(\widetilde{D}, \lambda) \in \operatorname{Diff}_{\Gamma}^{1}\left(\partial \widetilde{M} ; \widetilde{E}_{\mid \partial \widetilde{M}}\right)$ is equal to the family of $\Gamma$-invariant differential operators on $\widetilde{E}_{0} \oplus \widetilde{E}_{0}$ given by

$$
\left(\begin{array}{cc}
0 & \widetilde{D}_{0}-i \lambda \\
\widetilde{D}_{0}+i \lambda & 0
\end{array}\right)
$$

Now, let $\not{D} \in \operatorname{Diff}_{b, \Lambda}^{1}(M ; \mathcal{E})$ denote the $\Lambda-b$-differential operator on $M$ induced by $\widetilde{D}$. We then define in the same way the $\Lambda$-boundary operator $\not{D}_{0} \in \operatorname{Diff}_{\Lambda}^{1}\left(\partial M ; \mathcal{E}_{0}\right)$ and the indicial family $I(\not \mathcal{D}, \lambda) \in \operatorname{Diff}_{\Lambda}^{1}\left(\partial M ; \mathcal{E}_{\mid \partial M}\right)$.

\section{Noncommutative Spectral Sections and the $\mathcal{B}^{\infty}$-pseudodifferential Calculus}

We first recall the general definition, given in [W1], of noncommutative spectral section. We adopt the notation in [LPi1] and thus change those of [W1] accordingly. We are given a unital $C^{*}$-algebra $A$ and a (full) Hilbert module $\mathcal{H}$ over $A$. We denote by $\mathrm{B}_{A}(\mathcal{H})\left(\operatorname{resp} . \mathrm{K}_{A}(\mathcal{H})\right)$ the bounded $A$ linear adjointable (resp. $A$-compact) endomorphisms of $\mathcal{H}$. We consider $\mathcal{D}$ a self-adjoint densely defined, $A$-linear, unbounded regular operator on $\mathcal{H}$ $([\mathrm{BJ}])$. One can define a functional calculus associated to any such operator $\mathcal{D}$; it is a $C^{*}$-homomorphism between the $C^{*}$-algebra $C([-\infty, \infty], \mathbb{C})$ of bounded continuous functions $f$ on $\mathbb{R}$ such that $f( \pm \infty)=\lim _{x \rightarrow \pm \infty} f(x)$ exist and the $C^{*}$-algebra of bounded $A$-endomorphism $\mathrm{B}_{A}(\mathcal{H})$. Thus 
$C([-\infty, \infty], \mathbb{C}) \ni f \rightarrow f(\mathcal{D}) \in \mathrm{B}_{A}(\mathcal{H})$. We shall denote by $C^{\infty}([-\infty, \infty], \mathbb{C})$ the smooth elements of $C([-\infty, \infty], \mathbb{C})$. The functional calculus is needed in order to rigorously encode the characteristic property of a spectral section (see formula (0.4)) of being the identity on large positive "eigenvalues" and zero on large negative "eigenvalues". To this end it is also useful to introduce the notion of smooth spectral cut; this is a smooth function $\chi \in C^{\infty}(\mathbb{R},[0,1])$ such that for some $s_{1}, s_{2} \in \mathbb{R}, s_{1}<s_{2}$, it satisfies

$$
\chi(t)= \begin{cases}0 & \text { if } t \leq s_{1} \\ 1 & \text { if } t \geq s_{2}\end{cases}
$$

Definition 2.1. Let $(\mathcal{H}, \mathcal{D})$ be as above. A self-adjoint projection $\mathcal{P}=$ $\mathcal{P}^{2}=\mathcal{P}^{*} \in \mathrm{B}_{A}(\mathcal{H})$ is a spectral section for $\mathcal{D}$ if there exist smooth spectral cuts $\chi_{1}, \chi_{2}$, with $\chi_{2}(t)=1 \forall t \in \operatorname{supp}\left(\chi_{1}\right)$, such that

$$
\chi_{1}(\mathcal{D})(\mathcal{H}) \subset \mathcal{P}(\mathcal{H}) \subset \chi_{2}(\mathcal{D})(\mathcal{H}) .
$$

We recall one of the main results of [W1]:

Theorem 2.2 [W1]. A spectral section for $\mathcal{D}$ as above exists if and only if $\operatorname{Ind}(\mathcal{D})=0$ in $K_{1}(A)$.

Let $\Gamma \rightarrow \tilde{N} \rightarrow N$ be a Galois covering as in the previous section. Let $F$ be a hermitian vector bundle over $N$. We are interested in the case in which the $C^{*}$-algebra $A$ is equal to $\Lambda \equiv C_{r}^{*}(\Gamma), \mathcal{H}=L_{\Lambda}^{2}(N, \mathcal{F})$, $\mathcal{F}=F \otimes\left(C_{r}^{*}(\Gamma) \times_{\Gamma} \tilde{N}\right)$ and $\mathcal{D}$ is a geometric $C_{r}^{*}(\Gamma)$-differential operator of order one.

If, in particular, $N=\partial M$ and $\mathcal{D}$ is equal to the boundary operator $\mathscr{D}_{0}$ (of $\not D$ ) considered at the end of sect. 1, then the following important result holds:

Proposition 2.3. The $K_{1}$-index of the boundary family $\not{D}_{0}$ is always zero: $\operatorname{Ind}\left(\mathcal{D}_{0}\right)=0$ in $K_{1}\left(C_{r}^{*}(\Gamma)\right)$.

Proof. The operator $\not{D}_{0}$ defines a class $\left[\not_{0}\right] \in K K^{1}\left(C^{0}(\partial M), C_{r}^{*}(\Gamma)\right)$. If $\pi^{\partial M}: \partial M \rightarrow$ pt denotes the mapping of $\partial M$ to a point, then, under the natural isomorphism $K K^{1}\left(\mathbb{C}, C_{r}^{*}(\Gamma)\right) \cong K_{1}\left(C_{r}^{*}(\Gamma)\right)$, we have $\operatorname{Ind}\left(\mathcal{D}_{0}\right)=$ $\pi_{*}^{\partial M}\left[\mathcal{D}_{0}\right]$. Let $C_{\partial M}^{0}(M) \subset C^{0}(M)$ denote the ideal of continuous functions on $M$ vanishing on the boundary, let $i$ be the natural inclusion of $\partial M$ into $M$ and consider the long exact sequence associated to $C_{\partial M}^{0}(M)$ and $C^{0}(M)$. We have, in particular, the exactness of

$$
\begin{aligned}
K K^{0}\left(C_{\partial M}^{0}(M), C_{r}^{*}(\Gamma)\right) \stackrel{\delta}{\longrightarrow} K K^{1}\left(C^{0}(\partial M), C_{r}^{*}(\Gamma)\right) \\
\stackrel{i_{*}}{\longrightarrow} K K^{1}\left(C^{0}(M), C_{r}^{*}(\Gamma)\right) .
\end{aligned}
$$


By assumption and by the results of [BaDoT] (see alternatively [MPi1]) we have $\left[\mathcal{D}_{0}\right]=\delta[\not \mathcal{D}]$ where $[\not \mathcal{D}] \in K K^{0}\left(C_{\partial M}^{0}(M), C_{r}^{*}(\Gamma)\right)$ is the class defined by the Dirac operator $\not D$ on $M$. If we denote by $\pi$ the mapping of $M$ to a point, then by functoriality $\pi_{*}^{\partial M}=\pi_{*} \circ i_{*}$. On the other hand from the exactness of $(2.2)$ we have $i_{*} \circ \delta=0$. Summarizing

$$
\operatorname{Ind}\left(\mathcal{D}_{0}\right)=\pi_{*}^{\partial M}\left[\not{D}_{0}\right]=\pi_{*}^{\partial M} \circ \delta[\not \mathcal{D}]=\pi_{*} \circ i_{*} \circ \delta[\not \mathcal{D}]=0,
$$

and the proposition follows.

According to Theorem 2.2 there always exists a noncommutative spectral section for the boundary operator $\not{D}_{0}$. Notice, however, that in the family case (i.e. for $C^{0}(B)$-Hilbert modules) a spectral section $P$ has two additional properties when compared to a spectral section as in Definition 2.1 ; it is given by a family of 0 -th order pseudodifferential operators which depends smoothly on the base variables. These two properties are crucial in the APS family index theory developed in [MPi2,3]. In the case of Galois coverings the first property translates naturally into the requirement that $\mathcal{P} \in \Psi_{\Lambda}^{0}(N, \mathcal{F})$. As far as the smoothness condition is concerned, we concentrate on the virtually nilpotent case as in [LPi1] and thus require that $\mathcal{P} \in \Psi_{\mathcal{B} \infty}^{0}\left(N, \mathcal{F}^{\infty}\right)$ with $\mathcal{F}^{\infty}=F \otimes\left(\mathcal{B}^{\infty} \times_{\Gamma} \widetilde{N}\right)$ (see $\left.(0.1)\right)$. As explained in $\left[\right.$ Lo1,2] the passage from $\Lambda$ to $\mathcal{B}^{\infty}$ should be precisely thought of as the passage to the smooth category, moreover it allows us to consider the Chern character of the relevant index classes with value in the noncommutative topological de Rham homology $\bar{H}_{*}\left(\mathcal{B}^{\infty}\right)$ (something much more interesting than $\left.\bar{H}_{*}(\Lambda)\right)$.

In order to deal with this smoothness property, we are going to recall Lott's correspondence between smooth $\mathcal{B}^{\infty}$-sections or operators on the base and associated smooth objects of rapid decay on the covering $\widetilde{N}$. Notice that $\widetilde{N}$ is not assumed to be connected. Let $R_{\gamma}$ denote the right action of $\gamma \in \Gamma$ on $\widetilde{N}$. First of all, any section $s \in C^{\infty}\left(N, \mathcal{F}^{\infty}\right)$ may be written as $s=\sum_{\gamma \in \Gamma} R_{\gamma}^{*}\left(s_{1}\right) \gamma$ where $s_{1}$ belongs to the Schwartz space $S(\widetilde{N}, \widetilde{F})$. Second, any $\mathcal{K}$ in $\Psi_{\mathcal{B}^{\infty}}^{-\infty}\left(N, \mathcal{F}^{\infty}\right)$ corresponds to a $\Gamma$-invariant smoothing operator $\widetilde{K}$ in $\Psi_{\Gamma}^{-\infty}(\widetilde{N}, \widetilde{F})$ in a such a way that

$$
\forall s_{1} \in S(\widetilde{N}, \widetilde{F}), \quad \mathcal{K}\left(\sum_{\gamma \in \Gamma} R_{\gamma}^{*}\left(s_{1}\right) \gamma\right)=\sum_{\gamma \in \Gamma} R_{\gamma}^{*}\left(\widetilde{K}\left(s_{1}\right)\right) \gamma
$$

moreover its Schwartz kernel, still denoted $\widetilde{K}$, is rapidly decreasing in the following sense: $\forall q \in \mathbb{N}^{*}$ and for all multi-indices $\alpha, \beta$

$$
\sup _{x, y \in \widetilde{N}}\left\|\left((1+d(x, y))^{q} \nabla_{x}^{\alpha} \nabla_{y}^{\beta} \widetilde{K}(x, y)\right)\right\|<+\infty,
$$


where $d(\cdot, \cdot)$ denotes the geodesic distance on $\widetilde{N}$. The distance $d(x, y)$ is well defined if $x, y$ belong to the same connected component of $\widetilde{N}$. If they belong to different connected components we fix a fundamental domain $F$ in $\widetilde{N}$ and set $d(x, y)=\left\|\gamma_{1}^{-1} \gamma_{2}\right\|$ with $x \gamma_{1}, y \gamma_{2} \in F$.

REMARK. Let $\widetilde{D}$ be a Dirac-type operator as in sect. 1 . If we express $\widetilde{N}$ as the disjoint union of its connected components, $\widetilde{N}=\bigsqcup_{\alpha} \widetilde{N}_{\alpha}$, then, obviously, $\widetilde{D}$ is block-diagonal with respect to the orthogonal decomposition $L^{2}(\widetilde{N}, \widetilde{F})=\bigoplus L^{2}\left(\widetilde{N}_{\alpha}, \widetilde{F}\right)$. This implies that for any $\phi \in C_{0}^{\infty}(\mathbb{R})$ and $s>0$, the Schwartz kernels of the operators $\phi(\widetilde{D}), \exp \left(-s \widetilde{I D}^{2}\right)$ are zero on $N_{\alpha} \times N_{\beta}$, $\alpha \neq \beta$.

From now on, we shall consider only Dirac-type operators $\not$ on a manifold $M$ [resp. N] with [resp. without] boundary.

Proposition 2.4. Let $\chi \in C^{\infty}(\mathbb{R} ; \mathbb{R})$ be a spectral cut and let $\Psi \in C_{0}^{\infty}(\mathbb{R} ; \mathbb{R})$.

(1) Let $\Gamma$ be any discrete finitely presented group. Let $\not{D} \in \operatorname{Diff}_{\Lambda}^{1}(N ; \mathcal{F})$ be a $\Lambda$-differential operator as in sect. 1 . Then $\chi(\not \mathcal{D})[$ resp. $\psi(\not \mathcal{D})]$ is well defined in $\Psi_{\Lambda}^{0}(N ; \mathcal{F})\left[\operatorname{resp} . \Psi_{\Lambda}^{-\infty}(N ; \mathcal{F})\right]$.

(2) If, moreover, $\Gamma$ is virtually nilpotent then $\chi(\not \mathcal{D})[$ resp. $\psi(\not \mathcal{D})]$ is well defined in $\Psi_{\mathcal{B}^{\infty}}^{0}\left(N ; \mathcal{F}^{\infty}\right)\left[\right.$ resp. $\left.\Psi_{\mathcal{B} \infty}^{-\infty}\left(N ; \mathcal{F}^{\infty}\right)\right]$.

REMARK. If in (2) $\Gamma$ is not virtually nilpotent, then it is not at all clear how to define $\chi(\not \mathcal{D})$ in $\Psi_{\mathcal{B}^{\infty}}^{0}\left(N ; \mathcal{F}^{\infty}\right)$ where $\mathcal{B}^{\infty}$ is any dense subalgebra of $\Lambda$ closed under holomorphic functional calculus. The problem can be seen already at the algebraic level: if $a=a^{*} \in \mathcal{B}^{\infty}$ then we see no reason why $\chi(a)$ should also belong to $\mathcal{B}^{\infty}$. This is one of the main reasons why we restrict ourselves to virtually nilpotent groups in dealing with the smoothness condition.

Proof. (1) The proof is an easy adaptation to the $\Lambda$-Hilbert module $L_{\Lambda}^{2}(N ; \mathcal{F})$ of the proof given in $[\mathrm{T}, \mathrm{p} .300]$. We leave the details to the reader.

(2) The operator associated to $\chi(\not \mathcal{D})$ on the covering is $\chi(\widetilde{D})$, it acts on $L^{2}(\widetilde{N} ; \widetilde{E})$. We are going to show that $\chi(\widetilde{D})$ is the sum of a smoothing operator (in the sense recalled above) and of a $\Gamma$-invariant pseudo-differential operator whose Schwartz kernel is $\varepsilon$-local on $\widetilde{N} \times \widetilde{N}$ for some $\varepsilon>0$. This will prove that $\chi(\not \mathcal{D}) \in \Psi_{\mathcal{B}^{\infty}}^{0}\left(N ; \mathcal{F}^{\infty}\right)$, we leave the study of $\Psi(\not \mathcal{D})$ to the reader.

We consider $\hat{\chi}(t)=\int_{-\infty}^{+\infty} \mathrm{e}^{-i t z} \chi(z) d z$ for $t \in \mathbb{R}$. Let $\Phi \in C_{0}^{\infty}(\mathbb{R} ; \mathbb{R})$ be such that $\Phi(t) \equiv 1$ on a neighborhood of $0 \in \mathbb{R}$. Since $\chi(t)=1$ [resp. 0] for $t \gg 0$ [resp. $t \ll 0]$ we see that $[1-\Phi(t)] \hat{\chi}(t)$ belongs to the Schwartz 
GAFA

space $\mathcal{S}(\mathbb{R} ; \mathbb{C})$. Moreover, we have

$$
\chi(\widetilde{D})=\int_{-\infty}^{+\infty} \mathrm{e}^{i t \tilde{D}} \Phi(t) \hat{\chi}(t) \frac{d t}{2 \pi}+\int_{-\infty}^{+\infty} \mathrm{e}^{i t \tilde{D}}[1-\Phi(t)] \hat{\chi}(t) \frac{d t}{2 \pi} .
$$

Finite propagation speed estimates $([\mathrm{R}, \mathrm{p} .60])$ show that for all $t \in \mathbb{R}$ and $x \in \widetilde{N}$, supp $\mathrm{e}^{i t} \widetilde{D}_{(x, y)}$ is contained in the ball $B(x,|t|)$ of radius $|t|$. Since moreover $[1-\Phi(t)] \hat{\chi}(t) \in \mathcal{S}(\mathbb{R}, \mathbb{C})$, a standard argument shows that $\int_{-\infty}^{+\infty} \mathrm{e}^{i t} \widetilde{D}_{[1-\Phi(t)] \hat{\chi}(t) \frac{d t}{2 \pi}}$ is a smoothing operator on $\tilde{N}$. Using equation (2.4) and the fact (proved in (1)) that $\chi(\not \mathcal{D}) \in \Psi_{\Lambda}^{0}(N ; \mathcal{F})$, we therefore see that $\int_{-\infty}^{+\infty} \mathrm{e}^{i t} \widetilde{D}_{\Phi}(t) \hat{\chi}(t) \frac{d t}{2 \pi}$ is the associated operator (on the covering) to an element of $\Psi_{\Lambda}^{0}(N ; \mathcal{F})$. Using the finite propagation speed property recalled above and the fact that $\Phi$ is smooth with compact support, we see that $\int_{-\infty}^{+\infty} \mathrm{e}^{i t} \widetilde{D}_{\Phi}(t) \hat{\chi}(t) \frac{d t}{2 \pi}$ is a $\Gamma$-invariant pseudo-differential operator on $\widetilde{N}$ whose Schwartz kernel is $\varepsilon$-local on $\widetilde{N} \times \widetilde{N}$ for $\varepsilon>$ diameter $(\operatorname{supp}(\Phi))$. Proposition 2.4 is thus proved.

Proposition 2.5. Let $\not{D}$ be as in Proposition 2.4. Let $\mathcal{P}$ be a spectral section for $\not D$ and $\chi_{1}, \chi_{2}$ be two spectral cuts as in Definition 2.1. Then we can find $\Psi \in C_{0}^{\infty}(\mathbb{R} ; \mathbb{R})$ such that

$$
\mathcal{P}-\chi_{1}(\not \mathcal{D})=\Psi(\not \mathcal{D}) \circ\left[\mathcal{P}-\chi_{1}(\not \mathcal{D})\right] \circ \Psi(\not \mathcal{D}) \in \Psi_{\Lambda}^{-\infty}(N ; \mathcal{F}) .
$$

Moreover $\mathcal{P} \in \Psi_{\Lambda}^{0}(N ; \mathcal{F})$.

Proof. By assumption we have $\operatorname{Im} \chi_{1}(\not \mathcal{D}) \subset \operatorname{Im} \mathcal{P} \subset \operatorname{Im} \chi_{2}(\not \mathcal{D})$ and $\mathcal{P}=\mathcal{P}^{2}=\mathcal{P}^{*}$. Let $\chi_{3}$ be a third spectral cut such that $\chi_{3} \equiv 1$ on a neighborhood of $\operatorname{supp} \chi_{2}$. Then, one checks easily that (cf. [W1, Remark 2.1])

$$
\mathcal{P} \circ \chi_{1}(\not \mathcal{D})=\chi_{1}(\not \mathcal{D}) \circ \mathcal{P}=\chi_{1}(\not \mathcal{D}), \quad \mathcal{P} \circ \chi_{3}(\not \mathcal{D})=\chi_{3}(\not \mathcal{D}) \circ \mathcal{P}=\mathcal{P} .
$$

Now let $\Psi \in C_{0}^{\infty}(\mathbb{R} ; \mathbb{R})$ be such that $\Psi \equiv 1$ on a neighborhood of the compact set $\operatorname{supp}\left(1-\chi_{1}\right) \chi_{3}$. We then have

$$
\begin{aligned}
(\operatorname{Id}-\Psi(\not \mathcal{D})) \circ\left(\mathcal{P}-\chi_{1}(\not \mathcal{D})\right) & =(\operatorname{Id}-\Psi(\not \mathcal{D})) \circ\left(\operatorname{Id}-\chi_{1}(\not \mathcal{D})\right) \circ \mathcal{P} \\
& =(\operatorname{Id}-\Psi(\not \mathcal{D})) \circ\left(\operatorname{Id}-\chi_{1}(\not{D})\right) \circ \chi_{3}(\not \mathcal{D}) \circ \mathcal{P}=0 .
\end{aligned}
$$

So, we have $\mathcal{P}-\chi_{1}(\not \mathcal{D})=\Psi(\not \mathcal{D}) \circ\left[\mathcal{P}-\chi_{1}(\not \mathcal{D})\right]$. Since $\mathcal{P}, \chi_{1}(\not{D}), \Psi(\not \mathcal{D})$ are all selfadjoint we obtain

$$
\mathcal{P}-\chi_{1}(\not \mathcal{D})=\left[\mathcal{P}-\chi_{1}(\not \mathcal{D})\right] \circ \Psi(\not \mathcal{D})=\Psi(\not \mathcal{D}) \circ\left[\mathcal{P}-\chi_{1}(\not \mathcal{D})\right] \circ \Psi(\not{D}) .
$$

A priori, $\mathcal{P}-\chi_{1}(\not \mathcal{D})$ is only a bounded adjointable operator on $L_{\Lambda}^{2}(N ; \mathcal{F})$, but Proposition $2.4(1)$ shows that $\Psi(\not \mathcal{D})$ and $\Psi(\not \mathcal{D}) \circ\left[\mathcal{P}-\chi_{1}(\not \mathcal{D})\right] \circ \Psi(\not \mathcal{D})$ belong 
to $\Psi_{\Lambda}^{-\infty}(N ; \mathcal{F})$ and that $\chi_{1}(\not \mathcal{D}) \in \Psi_{\Lambda}^{0}(N ; \mathcal{F})$. We therefore get immediately Proposition 2.5.

We shall need the following (partial) extension of Theorem 2.2:

Theorem 2.6. Let $\widetilde{D} \in \operatorname{Diff}_{\Gamma}^{1}(\widetilde{N}, \widetilde{F})$ be elliptic and formally self-adjoint. Let $\not{D} \in \operatorname{Diff}_{\Lambda}^{1}(N, \mathcal{F})$ be the associated $\Lambda$-differential operator.

(1) There exists a spectral section $\mathcal{P} \in \Psi_{\Lambda}^{0}(N, \mathcal{F})$ for $\not D$ if and only if $\operatorname{Ind}(\not D)=0$ in $K_{1}\left(C_{r}^{*}(\Gamma)\right)$.

(2) If moreover $\Gamma$ is virtually nilpotent then $\mathcal{P}$ can be chosen in $\Psi_{\mathcal{B}^{\infty}}^{0}\left(N, \mathcal{F}^{\infty}\right)$.

Proof. Part (1) is an immediate consequence of Proposition 2.5 and the Theorem 2.2. Part (2) of Theorem 2.6 will be proved at the end this section.

If $\Gamma$ is virtually nilpotent then we shall always assume a spectral section $\mathcal{P}$ to be in the $\mathcal{B}^{\infty}$-pseudodifferential calculus $\mathcal{P} \in \Psi_{\mathcal{B}^{\infty}}^{0}\left(N, \mathcal{F}^{\infty}\right)$.

As a consequence of Theorem 2.6 and Proposition 2.3 we immediately obtain

Theorem 2.7. Let $\Gamma \rightarrow \widetilde{M} \rightarrow M$ be a Galois covering with boundary, let $\widetilde{D} \in \operatorname{Diff}_{b, \Gamma}^{1}(\widetilde{M}, \widetilde{E})$ be a $\Gamma$-invariant Dirac operator and let $\not{D}$ be the associated $\Lambda$-differential operator.

(1) There always exists a spectral section $\mathcal{P} \in \Psi_{\Lambda}^{0}\left(\partial M, \mathcal{E}_{0}\right)$ for the boundary operator $\mathcal{D}_{0} \in \operatorname{Diff}_{\Lambda}^{1}\left(\partial M, \mathcal{E}_{0}\right)$.

(2) If $\Gamma$ is virtually nilpotent then $\mathcal{P}$ can be chosen in $\Psi_{\mathcal{B}^{\infty}}^{0}\left(\partial M, \mathcal{E}_{0}^{\infty}\right)$.

Let $\not D \in \operatorname{Diff}_{\Lambda}^{1}(M, \mathcal{F})$ be elliptic and self-adjoint. If one spectral section exists then there are infinitely many. More precisely we have the following proposition and its corollary.

Proposition 2.8. (1) If a spectral section $\mathcal{P}$ for $\not \mathcal{D}$ exists then for any smooth spectral cut $\chi_{1}$ there exists a smooth spectral cut $\chi_{2}$, with $\chi_{1}(t) \chi_{2}(t)$ $=\chi_{1}(t)$, and a spectral section $\mathcal{R}$ satisfying (2.1). Similarly for any spectral cut $\chi_{2}$ there exists a $\chi_{1}$, with $\chi_{1}(t) \chi_{2}(t)=\chi_{1}(t)$, and a spectral section $\mathcal{Q}$ satisfying (2.1).

(2) If $\Gamma$ is virtually nilpotent then $\mathcal{Q}, \mathcal{R}$ can be chosen in $\Psi_{\mathcal{B}^{\infty}}^{0}\left(N, \mathcal{F}^{\infty}\right)$

Corollary 2.9. (1) Let $\mathcal{P}_{i}, i=1,2$, be spectral sections for $\not{D}$. Then there exist spectral sections $\mathcal{R}, \mathcal{Q}$ such that, for $i=1,2$,

$$
\mathcal{P}_{i} \circ \mathcal{R}=\mathcal{R} \circ \mathcal{P}_{i}=\mathcal{P}_{i}, \quad \mathcal{P}_{i} \circ \mathcal{Q}=\mathcal{Q} \circ \mathcal{P}_{i}=\mathcal{Q} .
$$

(2) If $\Gamma$ is virtually nilpotent then $\mathcal{R}, \mathcal{Q}$ can be chosen in $\Psi_{\mathcal{B}^{\infty}}^{0}\left(N, \mathcal{F}^{\infty}\right)$. 
Part (1) of both statements is a consequence of Proposition 2.5 and Proposition (2.5) of [W1]. Part (2) can then be proved using the argument given in the proof of part (4) of the next proposition. We leave the easy details to the reader.

Suppose for a moment that $\not \mathcal{D}$ is invertible; we obviously have $\operatorname{Ind}(\not{D})=0$ in $K_{1}\left(C_{r}^{*}(\Gamma)\right)$. In this case we have a canonical spectral section $\Pi_{\geq}$simply given by the projection onto the positive part of $\not{D}$

$$
\Pi_{\geq}=\frac{1}{2}\left(\frac{\not \mathcal{D}}{|\not{D}|}+1\right) .
$$

Assume now that $\not D$ is not invertible but that $\operatorname{Ind}(\not \mathcal{D})=0$ in $K_{1}\left(C_{r}^{*}(\Gamma)\right)$. Let $\mathcal{P} \in \Psi_{\Lambda}^{0}(N, \mathcal{F})$ be a spectral section for $\not{D}$. The next proposition explains how $\mathcal{P}$ provides a specific trivialization of the index class $\operatorname{Ind}(\not \mathcal{D})$, moreover its part (4) implies Theorem 2.6 (2).

Proposition 2.10. Let $\mathcal{P}$ be a spectral section for $\not{D}$. Then there exists a self-adjoint operator $\mathcal{A}_{\mathcal{P}}^{0} \in \Psi_{\Lambda}^{-\infty}(N, \mathcal{F})$ with the following properties:

(1) We can find $R>0$ such that $\psi(\not \mathcal{D}) \circ \mathcal{A}_{\mathcal{P}}^{0} \equiv 0$ for any smooth function $\psi \in C^{\infty}([-\infty, \infty], \mathbb{C})$ vanishing on $[-R, R]$.

(2) $\not{D}+\mathcal{A}_{\mathcal{P}}^{0}$ is invertible.

(3) $\mathcal{P}$ is equal to the projection onto the positive part of $\not{D}+\mathcal{A}_{\mathcal{P}}^{0}$,

$$
\mathcal{P}=\frac{1}{2}\left(\frac{\not \mathcal{D}+\mathcal{A}_{\mathcal{P}}^{0}}{\left|\not{D}+\mathcal{A}_{\mathcal{P}}^{0}\right|}+1\right) .
$$

(4) If moreover $\Gamma$ is virtually nilpotent then we can find a selfadjoint operator $\mathcal{A}^{0} \in \Psi_{\mathcal{B} \infty}^{-\infty}\left(N, \mathcal{F}^{\infty}\right)$ satisfying property (1) above, such that $\not{D}+\mathcal{A}^{0}$ is invertible. Furthermore $\frac{1}{2}\left(\frac{\not \mathcal{D}+\mathcal{A}^{0}}{\left|\not{D}+\mathcal{A}^{0}\right|}+1\right)$ is a spectral section belonging to $\Psi_{\mathcal{B}^{\infty}}^{0}\left(N ; \mathcal{F}^{\infty}\right)$.

Proof. We show parts (1),(2),(3) and (4) at the same time. We follow closely the proof of the Lemma 2.7 of [W1] (which is inspired by [MPi2]). Let us choose $\delta>0$ such that for any smooth spectral cuts $\chi_{-}, \chi_{+}$with $\chi_{+}(t)=1$ for $t \geq-\delta, \chi_{-}(t)=0$ for $t \leq \delta$; we have

$$
\operatorname{Im} \chi_{-}(\not D) \subset \operatorname{Im} \mathcal{P} \subset \operatorname{Im} \chi_{+}(\not \mathcal{D}) .
$$

As in [W1] we can find two spectral sections $\mathcal{Q}$ and $\mathcal{R}$ such that, with respect to the orthogonal decomposition of $L_{\Lambda}^{2}(N, \mathcal{F})$ induced by the four projections $\mathcal{Q}, \mathrm{Id}-\mathcal{R}, \mathcal{P} \mathcal{R}(\operatorname{Id}-\mathcal{Q}),(\mathrm{Id}-\mathcal{P}) \mathcal{R}(\mathrm{Id}-\mathcal{Q})$, the following operator,

$$
\widehat{\mathcal{D}}=\mathcal{Q} \not \mathcal{Q}+\mathcal{P} \mathcal{R}(\mathrm{Id}-\mathcal{Q})+(\operatorname{Id}-\mathcal{R}) \not \mathcal{D}(\mathrm{Id}-\mathcal{R})-(\operatorname{Id}-\mathcal{P}) \mathcal{R}(\operatorname{Id}-\mathcal{Q})
$$


is block diagonal, invertible and has the property that $\mathcal{P}$ is the projection onto its positive part. Using standard arguments one can find $\Psi_{1} \in$ $C_{0}^{\infty}(\mathbb{R} ; \mathbb{R})$ in such a way that the operator

$$
\mathcal{A}_{\mathcal{P}}^{0}=\widehat{\mathscr{D}}-\not{D}=\Psi_{1}(\not{D}) \circ[\widehat{\mathscr{D}}-\not{D}] \circ \Psi_{1}(\not{D})
$$

belongs to $\Psi_{\Lambda}^{-\infty}(N, \mathcal{F})$. Moreover, using the density of $\Psi_{\mathcal{B}^{\infty}}^{-\infty}\left(N, \mathcal{F}^{\infty}\right)$ in $\Psi_{\Lambda}^{-\infty}(N, \mathcal{F})$ for the $L_{\Lambda}^{2}$-operator norm, it is easy to find an operator $\mathcal{A}^{0} \in$ $\Psi_{\mathcal{B}}^{-\infty}\left(N, \mathcal{F}^{\infty}\right)$ "close to" $\mathcal{A}_{\mathcal{P}}^{0}$ satisfying all the properties of part (4). Proposition 2.10 is therefore proven.

REMARK. The proof of (4) shows that if we start with $\mathcal{P} \in \Psi_{\mathcal{B}^{\infty}}^{0}$ then the corresponding $\mathcal{A}_{\mathcal{P}}$ will belong to $\Psi_{\mathcal{B} \infty}^{-\infty}$.

Remark. In the family APS index theory developed in [MPi2,3] Proposition 2.10 is of great importance. Applied to the boundary family $\not_{0}$ allows us to reduce the index problem associated to an arbitrary spectral section $P$ to the index problem for the canonical spectral section $\Pi_{\geq}$but for a larger class of operators. See the beginning of sect. 4 for more informations on this fundamental point.

It is clear from the proof of Proposition 2.10 that the operator $\mathcal{A}_{\mathcal{P}}^{0}$ is not unique.

Definition 2.11. We say that $\mathcal{A}_{\mathcal{P}}^{0} \in \Psi_{\Lambda}^{-\infty}(N, \mathcal{F})$ is a $\mathcal{P}$-trivializing operator for $\mathcal{D}$ if it satisfies the first three properties stated in Proposition 2.10. If $\Gamma$ is virtually nilpotent we shall always require a $\mathcal{P}$-trivializing operator to belong to $\Psi_{\mathcal{B} \infty}^{-\infty}\left(N, \mathcal{F}^{\infty}\right)$.

\section{$3 \quad \mathcal{P}$-higher Eta Invariants}

In this section we assume that $\Gamma$ is virtually nilpotent. Generalizing to the noncommutative case the eta form of Bismut-Cheeger, Lott has introduced in [Lo2] the notion of higher eta invariant for an $L^{2}$-invertible $\Gamma$-invariant Dirac operator $\widetilde{D} \in \operatorname{Diff}_{\Gamma}^{1}(\widetilde{N}, \widetilde{F})$. Later, the higher eta invariant was defined in [LPi1] under the weaker assumption: $\exists \delta>0$ such that $\operatorname{spec}(\widetilde{D D}) \cap]-\delta, \delta[=\{0\}$.

In the general case we need to use the notion of noncommutative spectral section. Thus, following the general philosophy, we want to pass from the (commutative) $\mathcal{P}$-eta form of [MPi2] to a (noncommutative) $\mathcal{P}$-higher eta invariant. 
Let $N$ be a Riemannian manifold. Let $\Gamma \rightarrow \widetilde{N} \rightarrow N$ be a Galois covering of odd dimension, let $\not D \in \operatorname{Diff}^{1}(N, E)$ be a Dirac-type operator, as in sect. 1 we consider $\widetilde{D} \in \operatorname{Diff}_{\Gamma}^{1}(\widetilde{N}, \widetilde{E})$ its lift to $\widetilde{N}$ and $E_{\sigma}=E \otimes \operatorname{Cl}(1)$, $\widetilde{E}_{\sigma}=\widetilde{E} \otimes \mathrm{Cl}(1)$. We denote by $\Upsilon$ the obvious grading on $E_{\sigma}=E \oplus E$; we will frequently identify an operator $U$ acting on the sections of the bundle $\widetilde{E}$ [resp. $\mathcal{E}$ ] to the operator $U \oplus U$ acting on the sections of the bundle $\widetilde{E}_{\sigma}$ [resp. $\left.\mathcal{E}_{\sigma}\right]$. Let $\not{D}$ be the associated $\mathcal{B}^{\infty}$-linear operator acting on $C^{\infty}\left(N, \mathcal{E}^{\infty}\right)$ as in sect. 1. Suppose that $\operatorname{Ind}(\not \mathcal{D})=0$ in $K_{1}\left(C_{r}^{*}(\Gamma)\right)$ and let $\mathcal{P} \in \Psi_{\mathcal{B}^{\infty}}^{0}\left(N, \mathcal{E}^{\infty}\right)$ be a spectral section for $\not{D}$. Let $\mathcal{A}_{\mathcal{P}}^{0} \in \Psi_{\mathcal{B} \infty}^{-\infty}\left(N, \mathcal{E}^{\infty}\right)$ be a $\mathcal{P}$-trivializing operator as in Proposition 2.10. We recall that for each $k \in \mathbb{N}, \widehat{\Omega}_{k}\left(\mathcal{B}^{\infty}\right)$ denotes the set of differential forms:

$$
\sum_{\gamma_{0}, \ldots, \gamma_{k} \in \Gamma} a_{\gamma_{0}, \ldots, \gamma_{k}} \gamma_{0} d \gamma_{1} \ldots d g_{k}
$$

where the family of complex numbers $a_{\gamma_{0}, \ldots, \gamma_{k}}$ is of rapid decay with respect to any power of $\left(\left\|\gamma_{0}\right\|+\cdots+\left\|\gamma_{k}\right\|\right)$, \|\| being a fixed word metric on $\Gamma$. Then, the topological graded differential algebra associated to $\mathcal{B}^{\infty}$ is defined to be $\widehat{\Omega}_{*}\left(\mathcal{B}^{\infty}\right)=\bigoplus_{k \in \mathbb{N}} \widehat{\Omega}_{k}\left(\mathcal{B}^{\infty}\right)$. Let $\varphi \in C^{\infty}(\mathbb{R}, \mathbb{R})$ with $\varphi(t)=0$ for $t<1$ and $\varphi(t)=1$ for $t>2$. We consider the noncommutative superconnection $\mathbb{B}_{s, \mathcal{P}}: C^{\infty}\left(N, \mathcal{E}_{\sigma}^{\infty}\right) \rightarrow C^{\infty}\left(N, \mathcal{E}_{\sigma}^{\infty} \otimes_{\mathcal{B}^{\infty}} \widehat{\Omega}_{*}\left(\mathcal{B}^{\infty}\right)\right)$ defined by

$$
\mathbb{B}_{s, \mathcal{P}}=\Upsilon \nabla+\sigma s\left(\not \mathcal{D}+\varphi(s) \mathcal{A}_{\mathcal{P}}^{0}\right) .
$$

(3.1) is a perturbation of Lott's superconnection considered in [Lo1,2]; it depends on the spectral section $\mathcal{P}$ through the $\mathcal{P}$-trivializing operator $\mathcal{A}_{\mathcal{P}}^{0}$.

REMARK. As pointed out by Connes, it is more convenient in noncommutative geometry to deal with right-modules. For this reason it is necessary to introduce the grading $\Upsilon$ in the definition of a noncommutative superconnection ([Co, p. 435); thus our sign rules differ slightly from those of Quillen [Q].

The definition of $\mathbb{B}_{s, \mathcal{P}}$ (and of Lott's superconnection) exploits the natural isomorphisms of Frechet spaces

$$
\begin{gathered}
\mathcal{S}(\widetilde{N}, \widetilde{E}) \otimes_{\mathcal{B} \infty} \widehat{\Omega}_{k}\left(\mathcal{B}^{\infty}\right) \rightarrow C^{\infty}\left(N, \mathcal{E}^{\infty} \otimes_{\mathcal{B}^{\infty}} \widehat{\Omega}_{k}\left(\mathcal{B}^{\infty}\right)\right) \quad \forall k \in \mathbb{N} \\
\sum_{\gamma_{1}, \ldots, \gamma_{k} \in \Gamma} f_{\gamma_{1}, \ldots, \gamma_{k}}(z) \otimes e d \gamma_{1} \ldots d \gamma_{k} \rightarrow \sum_{\gamma_{0}, \gamma_{1}, \ldots, \gamma_{k} \in \Gamma} R_{\gamma_{0}}^{*}\left(f_{\gamma_{1}, \ldots, \gamma_{k}}\right)(z) \gamma_{0} d \gamma_{1} \ldots d \gamma_{k}
\end{gathered}
$$

where $e$ is the neutral element of $\Gamma$ and for any fixed point $z_{0} \in \tilde{N}$, the $f_{\gamma_{1}, \ldots, \gamma_{k}}(z)$ belong to the Schwartz space $\mathcal{S}(\widetilde{N}, \widetilde{E})$ and are of rapid decay, together with all their covariant derivatives, with respect to $d\left(z, z_{0}\right)+\left\|\gamma_{1}\right\|+$ 
$\cdots\left\|\gamma_{k}\right\|$. Under this isomorphism the superconnection (3.1) corresponds to the operator

$$
\mathbb{B}_{s, \mathcal{P}}=\Upsilon \nabla+\sigma s\left(\widetilde{D}+\varphi(s) \widetilde{A}_{P}^{0}\right): \mathcal{S}\left(\widetilde{N}, \widetilde{E}_{\sigma}\right) \rightarrow \mathcal{S}\left(\widetilde{N}, \widetilde{E}_{\sigma}\right) \otimes_{\mathcal{B}^{\infty}} \widehat{\Omega}_{*}\left(\mathcal{B}^{\infty}\right) .
$$

Notice that we shall keep the same notation for these two descriptions of the perturbed Lott superconnection.

Now, let us consider a right $\widehat{\Omega}_{*}\left(\mathcal{B}^{\infty}\right)$-linear continuous endomorphism of $C^{\infty}\left(N, \mathcal{E}_{\sigma}^{\infty} \otimes_{\mathcal{B}^{\infty}} \widehat{\Omega}_{*}\left(\mathcal{B}^{\infty}\right)\right), \mathcal{K}$; for notational convenience assume that $\mathcal{K}$ sends sections of $\mathcal{E}_{\sigma}^{\infty}$ into sections of $\mathcal{E}_{\sigma}^{\infty} \otimes_{\mathcal{B}^{\infty}} \widehat{\Omega}_{k}\left(\mathcal{B}^{\infty}\right)$ for a fixed $k$. According to (3.2) the operator $\mathcal{K}$ corresponds to a distribution Schwartz kernel $\left(z, z^{\prime}\right) \rightarrow \widetilde{K}\left(z, z^{\prime}\right)$ on $\widetilde{N} \times \widetilde{N}$ of the form

$$
\widetilde{K}\left(z, z^{\prime}\right)=\sum_{\gamma_{1}, \ldots, \gamma_{k}} \widetilde{K}_{\gamma_{1}, \ldots, \gamma_{k}}\left(z, z^{\prime}\right) e d \gamma_{1} \ldots d \gamma_{k}
$$

such that $\forall f \in \mathcal{S}\left(\widetilde{N}, \widetilde{E}_{\sigma}\right)$

$$
\mathcal{K}\left(\sum_{\gamma \in \Gamma} R_{\gamma}^{*}(f) \gamma\right)=\sum_{\gamma_{0}, \gamma_{1}, \ldots, \gamma_{k}} \int_{\widetilde{N}} \widetilde{K}_{\gamma_{1}, \ldots, \gamma_{k}}\left(z \cdot \gamma_{0}, z^{\prime}\right) f\left(z^{\prime}\right) d z^{\prime} \gamma_{0} d \gamma_{1} \ldots d \gamma_{k}
$$

Fundamental examples of such operators on the covering are precisely given by $\Upsilon \nabla+\sigma s\left(\widetilde{D}+\varphi(s) \widetilde{A}_{P}^{0}\right), \Upsilon \nabla \widetilde{D}+\widetilde{D} \Upsilon \nabla, \Upsilon \nabla \widetilde{A}_{P}^{0}+\widetilde{A}_{P}^{0} \Upsilon \nabla$.

We are going to recall the decay property (DP), essentially introduced in $[$ Lo1,2] (see also [LPi1]), which gives a necessary and sufficient condition on $\widetilde{K}$ for $\mathcal{K}$ to be smoothing.

Definition 3.1. Let $F$ be a fundamental domain for the covering $\widetilde{N}$. Let $\mathcal{K}$ be a right $\widehat{\Omega}_{*}\left(\mathcal{B}^{\infty}\right)$-linear operator as above and let us assume that the Schwartz kernels $\widetilde{K}_{\gamma_{1}, \ldots, \gamma_{k}}$ in equation (3.4) are all $C^{\infty}$. Then we shall say that $\mathcal{K}($ or $\widetilde{K}$ ) satisfies the decay property $(D P)$ if for any $M \in \mathbb{N}$ and any multi-index of derivation $\alpha$ with respect to $\left(z, z^{\prime}\right)$ the supremum $C(\mathcal{K}, M, \alpha)$ of the set

$$
\begin{gathered}
\left\{\left[d(z, F)+\left\|\gamma_{1}\right\|+\cdots+\left\|\gamma_{k-1}\right\|+d\left(z^{\prime}, R_{\gamma_{k}}(F)\right)\right]^{M}\left|\nabla^{\alpha} \widetilde{K}_{\gamma_{1}, \ldots, \gamma_{k}}\left(z, z^{\prime}\right)\right|,\right. \\
\text { such that } \left.\gamma_{1}, \ldots, \gamma_{k} \in \Gamma, \quad\left(z, z^{\prime}\right) \in \widetilde{N}^{2}\right\}
\end{gathered}
$$

is finite.

Here the distance function $d(\cdot, \cdot)$ introduced after $(2.3)$ has been used.

Proposition 3.2. Let $\mathcal{K}$ be an in (3.5). Then $\mathcal{K}$ is smoothing in the $\mathcal{B}^{\infty}$ calculus if and only if $\widetilde{K}$ given by (3.4), (3.5) is smooth and satisfies the decay property (DP) of Definition 3.1. 
Definition 3.3. Let $s \rightarrow F(s)$ be a positive function on $\mathbb{R}^{+*} \equiv(0, \infty)$. Let $(\mathcal{K}(s))_{s \in \mathbb{R}^{+*}}$ be a family of right $\widehat{\Omega}_{*}\left(\mathcal{B}^{\infty}\right)$-linear operators as in Definition 3.1 above. We shall say that the $\mathcal{K}(s)$ (or $\widetilde{K}(s)$ ) satisfy the decay property $(D P)$ with respect to $F(s), s$ being $>0$, if for any $M \in \mathbb{N}$ and any multi-index of derivation $\alpha$ with respect to $\left(z, z^{\prime}\right)$ we can find a constant $D(M, \alpha)$ such that

$$
\forall s>0, \quad C(\mathcal{K}(s), M, \alpha) \leq D(M, \alpha) F(s) .
$$

The next proposition shows that this (DP) property is "preserved" under composition.

Proposition 3.4. Let $(\mathcal{K}(s))_{s>0}$ be a family of right $\widehat{\Omega}_{*}\left(\mathcal{B}^{\infty}\right)$-linear operators, as in Definition 3.1, satisfying property $(D P)$ uniformly with respect to $F(s), s$ being $>0$. Let $\mathcal{A}$ be a right $\widehat{\Omega}_{*}\left(\mathcal{B}^{\infty}\right)$-linear operator sending continuously the sections of $\mathcal{E}^{\infty}$ into those of $\mathcal{E}^{\infty} \otimes_{\mathcal{B}^{\infty}} \widehat{\Omega}_{m}\left(\mathcal{B}^{\infty}\right)$, where $m$ is a fixed non-negative integer. Then the operators $\mathcal{K}(s) \circ \mathcal{A}, \mathcal{A} \circ \mathcal{K}(s)$ will also satisfy property $(\underset{D P}{ })$ uniformly with respect to $F(s), s$ being $>0$, provided the operator $\widetilde{A}$ associated to $\mathcal{A}$ on the covering fulfills at least one of the following assumptions:

(1) $\widetilde{A}$ satisfies property (DP).

(2) $\widetilde{A}$ belongs to $\left\{\Upsilon \nabla \widetilde{D}+\widetilde{D} \Upsilon \nabla, \nabla^{2}, \Upsilon \nabla \widetilde{A}_{P}^{0}+\widetilde{A}_{P}^{0} \Upsilon \nabla\right\}$.

(3) $\widetilde{A}$ is a $\Gamma$-invariant pseudo-differential operator of any order acting on $L^{2}(\widetilde{N}, \widetilde{E})$ whose Schwartz kernel $\widetilde{A}\left(z, z^{\prime}\right)$ is $C^{\infty}$ outside the diagonal and of rapid decay (with all its derivatives) when $d\left(z, z^{\prime}\right) \rightarrow+\infty$.

We refer to [LPi1] for more details on the last two propositions.

Now we consider an operator $\widetilde{K}$ sending $S\left(\widetilde{N}, \widetilde{E}_{\sigma}\right)$ into $S\left(\widetilde{N}, \widetilde{E}_{\sigma}\right) \otimes_{\mathcal{B} \infty}$ $\widehat{\Omega}_{k}\left(\mathcal{B}^{\infty}\right)$ and satisfying the decay property (DP) as in Definition 3.1. We are going to recall how one can define (cf. [Lo1], [LPi1]) its supertrace in $\widehat{\widehat{\Omega}}_{*}\left(\mathcal{B}^{\infty}\right)$ (recall that this is the quotient of $\widehat{\Omega}_{*}\left(\mathcal{B}^{\infty}\right)$ by the closure of the space of graded commutators). Thus we consider the following $\widehat{\Omega}_{*}\left(\mathcal{B}^{\infty}\right)$ bundle over $N$ :

$$
\mathcal{E}_{\sigma}^{\infty} \otimes_{\mathcal{B}^{\infty}} \widehat{\Omega}_{*}\left(\mathcal{B}^{\infty}\right)=E_{\sigma} \otimes_{\mathbb{C}}\left(\tilde{N} \times_{\Gamma} \mathcal{B}^{\infty}\right) \otimes_{\mathcal{B}} \infty \widehat{\Omega}_{*}\left(\mathcal{B}^{\infty}\right) .
$$

We can find a finite open cover $\mathcal{U}=\left\{U_{j}, 1 \leq j \leq q\right\}$ of $N$ and associated trivializations of this bundle

$$
M_{j}:\left(\mathcal{E}_{\sigma}^{\infty} \otimes_{\mathcal{B} \infty} \widehat{\Omega}_{*}\left(\mathcal{B}^{\infty}\right)\right)_{\mid U_{j}} \rightarrow U_{j} \times\left(\mathbb{C}^{\operatorname{dim} E_{\sigma}} \otimes_{\mathbb{C}} \widehat{\Omega}_{*}\left(\mathcal{B}^{\infty}\right)\right),
$$

where $M_{j}$ is a right $\widehat{\Omega}_{*}\left(\mathcal{B}^{\infty}\right)$-linear bundle isomorphism. Next, let $\mathcal{K}$ denote the right $\widehat{\Omega}_{*}\left(\mathcal{B}^{\infty}\right)$-linear continuous endomorphism of $C^{\infty}\left(N, \mathcal{E}_{\sigma}^{\infty} \otimes_{\mathcal{B}}\right.$ 
$\left.\widehat{\Omega}_{*}\left(\mathcal{B}^{\infty}\right)\right)$ associated with $\widetilde{K}$. It is defined by a distribution Schwartz kernel, $\mathcal{K}\left(z, z^{\prime}\right) \in \operatorname{Hom}_{\widehat{\Omega}_{*}\left(\mathcal{B}^{\infty}\right)}\left[\left(\mathcal{E}_{\sigma}^{\infty}\right)_{z^{\prime}} \otimes_{\mathcal{B}^{\infty}} \widehat{\Omega}_{*}\left(\mathcal{B}^{\infty}\right),\left(\mathcal{E}_{\sigma}^{\infty}\right)_{z} \otimes_{\mathcal{B}^{\infty}} \widehat{\Omega}_{*}\left(\mathcal{B}^{\infty}\right)\right]$.

Thus for any section $u$ of $\mathcal{E}_{\sigma}^{\infty} \otimes_{\mathcal{B}^{\infty}} \widehat{\Omega}_{*}\left(\mathcal{B}^{\infty}\right)$ we can write, with a common abuse of notation,

$$
\forall z \in N, \quad \mathcal{K}(u)(z)=\int_{N} \mathcal{K}\left(z, z^{\prime}\right) u\left(z^{\prime}\right) d g\left(z^{\prime}\right),
$$

where $d g\left(z^{\prime}\right)$ is the Riemannian density on $N$. We are going to describe locally this Schwartz kernel. Let $U_{i}, U_{j}$ be two open subsets belonging to $\mathcal{U}$. Then we can find a finite number of distributions on $U_{i} \times U_{j}:\left(z, z^{\prime}\right) \rightarrow$ $\omega_{l}\left(z, z^{\prime}\right), 1 \leq l \leq m$ with values in $\widehat{\Omega}_{k}\left(\mathcal{B}^{\infty}\right)$ and also endomorphisms $A_{l} \in$ $\operatorname{End}\left(\mathbb{C}^{\operatorname{dim} E_{\sigma}}\right), 1 \leq l \leq m$, such that for any vector $s \in \mathbb{C}^{\operatorname{dim} E_{\sigma}}$ and for any $\alpha \in C_{\mathrm{comp}}^{\infty}\left(U_{i}, \widehat{\Omega}_{*}\left(\mathcal{B}^{\infty}\right)\right)$ we have

$$
\left[\mathcal{K} \circ M_{i}^{-1}(s \otimes \alpha)\right]_{\mid U_{j}}=M_{j}^{-1}\left[\sum_{l=1}^{m} A_{l}(s) \otimes\left(\int_{U_{i}} \omega_{l}\left(z, z^{\prime}\right) \alpha\left(z^{\prime}\right) d g\left(z^{\prime}\right)\right)\right] .
$$

Let $x \in N$; for any endomorphism $u \in\left(E_{\sigma}\right)_{x}$ we set:

$$
\operatorname{Str}_{\mathrm{Cl}(1)}=\frac{1}{2} \operatorname{Str}\left(\begin{array}{cc}
0 & 1 \\
-1 & 0
\end{array}\right) \circ u \text {. }
$$

Now we use the notation of equation (3.6) with $U_{i}=U_{j}$ a neighborhood of $x$. We set

$$
\operatorname{Str}_{\mathrm{Cl}(1)}(\mathcal{K}(x, x))=\sum_{l=1}^{m} \operatorname{Str}_{\mathrm{Cl}(1)}\left((-\Upsilon)^{\partial \omega_{l}} A_{l}\right) \omega_{l}(x, x)
$$

where $\Upsilon$ denotes the grading of $\mathbb{C}_{\sigma}^{\operatorname{dim} E}$; this differential form is intrinsically defined in $\widehat{\widehat{\Omega}}_{*}\left(\mathcal{B}^{\infty}\right)$. The minus sign in (3.7) will provide a definition of the higher eta-invariant so that Proposition 7.2 will hold true. Then we define the supertrace of $\widetilde{K}$ to be

$$
\operatorname{STR}_{\mathrm{Cl}(1)} \mathcal{K}=\int_{N} \operatorname{Str}_{\mathrm{Cl}(1)} \mathcal{K}(x, x) d g(x) \in \overline{\widehat{\Omega}}_{*}\left(\mathcal{B}^{\infty}\right),
$$

where $d g(x)$ is the Riemannian density of $N$. The next proposition comes from [Lo1] (see also [LPi1, Sect. 2]) and gives a precise formula for the supertrace of an operator satisfying the property (DP).

Proposition 3.5. Let $\Phi \in C_{0}^{\infty}(\tilde{N})$ be such that $\sum_{\gamma \in \Gamma} R_{\gamma}^{*}(\Phi) \equiv 1$. Let $(\mathcal{K}(s))_{s>0}$ be the family of right $\widehat{\Omega}_{*}\left(\mathcal{B}^{\infty}\right)$-linear operators considered in Definition 3.3 satisfying the decay property $(D P)$ uniformly with respect to 
$F(s), s$ being $>0$. Then, for any $s>0$, the supertrace of $\mathcal{K}(s)$ is given by

$$
\begin{aligned}
& \operatorname{STR}_{\mathrm{Cl}(1)} \mathcal{K}(s) \\
& \quad=\sum_{\gamma_{0}, \gamma_{1}, \ldots, \gamma_{k} \in \Gamma} \int_{\widetilde{M}} \Phi(z) \operatorname{Str}_{\mathrm{Cl}(1)} \widetilde{K}_{\gamma_{1}, \ldots, \gamma_{k}}\left(z \gamma_{0}, z\right) d z \gamma_{0} d \gamma_{1} \ldots d \gamma_{k} \in \bar{\Omega}_{*}\left(\mathcal{B}^{\infty}\right) .
\end{aligned}
$$

Moreover, for every semi-norm $P_{\text {semi }}$ of $\widehat{\widehat{\Omega}}_{*}\left(\mathcal{B}^{\infty}\right)$, we can find a real constant $C>0$ such that

$$
\forall s \in \mathbb{R}^{+*}, \quad P_{\text {semi }}\left(\operatorname{STR}_{\mathrm{Cl}(1)} \mathcal{K}(s)\right) \leq C F(s) .
$$

Using Duhamel's expansion it is possible to define the heat-kernel associated to the superconnection $\mathbb{B}_{s, \mathcal{P}}^{2}$, more explanations will be given in the proof of Theorem 3.4. It is a right $\widehat{\Omega}_{*}\left(\mathcal{B}^{\infty}\right)$-linear operator satisfying the decay property (DP) of Definition 3.1.

We can now state the main result of this section. First we recall our data and assumptions. We are assuming the Galois covering $\Gamma \rightarrow \widetilde{N} \rightarrow N$ is of odd dimension and the group $\Gamma$ of polynomial growth; we are considering a Dirac-type operator $\widetilde{D} \in \operatorname{Diff}_{\Gamma}^{1}(\widetilde{N}, \widetilde{E})$ and the associated $\Lambda$-differential operator $\not{D} \in \operatorname{Diff}_{\Lambda}^{1}(N, \mathcal{E})$. We make the fundamental assumption that $\operatorname{Ind}(\not \mathcal{D})=0$ in $K_{1}(\Lambda), \Lambda \equiv C_{r}^{*}(\Gamma)$. Let $\mathcal{P} \in \Psi_{\mathcal{B}^{\infty}}^{0}\left(N, \mathcal{E}^{\infty}\right)$ be a spectral section for $\not D$ and let $\mathcal{A}_{\mathcal{P}}^{0} \in \Psi_{\mathcal{B} \infty}^{-\infty}\left(N, \mathcal{E}^{\infty}\right)$ be a $\mathcal{P}$-trivializing operator as in Proposition 2.10. Let $\widetilde{A}_{P}^{0}$ the $\Gamma$-invariant rapidly decreasing smoothing operator associated to $\mathcal{A}_{\mathcal{P}}^{0}$.

Theorem 3.6. (1) Let $\mathbb{B}_{s, \mathcal{P}}$ be the superconnection $\Upsilon \nabla+\sigma s\left(\widetilde{\not D}+\varphi(s) \widetilde{A}_{P}^{0}\right)$. The following integral is absolutely convergent and well defined as an element of $\widehat{\widehat{\Omega}}_{*}\left(\mathcal{B}^{\infty}\right)$ :

$$
\widehat{\eta}_{\mathcal{P}} \equiv \frac{2}{\sqrt{\pi}} \int_{0}^{+\infty} \operatorname{STR}_{\mathrm{Cl}(1)}\left[\frac{d \mathbb{B}_{s, \mathcal{P}}}{d s} \exp \left(-\left(\mathbb{B}_{s, \mathcal{P}}^{2}\right)\right)\right] d s .
$$

(2) Modulo exact forms the integral (3.8) depends only on the spectral section $\mathcal{P}$ and not on $\varphi$ or the particular choice of the $\mathcal{P}$-trivializing operator $\mathcal{A}_{\mathcal{P}}^{0}$. It defines the $\mathcal{P}$-higher eta invariant as an element $\widehat{\eta}_{\mathcal{P}} \in$ $\widehat{\widehat{\Omega}}_{*}\left(\mathcal{B}^{\infty}\right) / d \widehat{\widehat{\Omega}}_{*}\left(\mathcal{B}^{\infty}\right)$.

Proof of Theorem 3.6. First we need to state two lemmas.

Lemma 3.7. (1) We can find a real $\delta>0$ such that for any real $\varepsilon_{1}>0$, for each $(a, q) \in \mathbb{N}^{2}$ we have

$$
\forall s>\varepsilon_{1}, \forall(x, y) \in \tilde{N}^{2},
$$




$$
\left|\left(\widetilde{D}+\widetilde{A}_{P}^{0}\right)^{a} \mathrm{e}^{-s^{2}\left(\widetilde{D}+\widetilde{A}_{P}^{0}\right)^{2}}(x, y)\right| \leq C_{q, a, \delta}[1+d(x, y)]^{-q} \mathrm{e}^{-\delta s^{2}},
$$

where $d(.,$.$) denotes the geodesic distance of the Riemannian manifold \tilde{N}$.

(2) Let $\widetilde{K}$ be a right $\widehat{\Omega}_{*}\left(\mathcal{B}^{\infty}\right)$-linear operator as in Definition 3.1 satis-

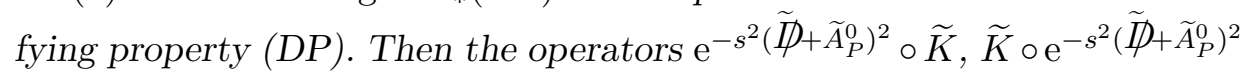
satisfy the decay property (DP) with respect to $1, s$ being in $] 0,1]$.

REMARK. Since $\widetilde{A}_{P}^{0}$ is not a differential operator we cannot use finite propagation speed estimates to prove point (1).

Lemma 3.8. The following integral is absolutely convergent:

$$
\frac{2}{\sqrt{\pi}} \int_{1}^{2} \operatorname{STR}_{\mathrm{Cl}(1)}\left(\frac{d}{d s}\left[s \sigma\left(\widetilde{D}+\varphi(s) \widetilde{A}_{P}^{0}\right)\right] \exp \left(-\mathbb{B}_{s, \mathcal{P}}^{2}\right)\right) d s .
$$

Let us assume Lemmas 3.7 and 3.8, which will proved in the next section, and let us prove the theorem. Let us recall that $\varphi(s) \equiv 0$ for $s \in[0,1]$. Then the convergence on $[0,1]$ of the integral (3.8) is a straightforward consequence of the local index theorem as pointed out in [Lo2, p. 219]. So, according to Lemma 3.8, we just have to prove the convergence on $[2,+\infty[$. We recall the equation (3.1) which defines $\mathbb{B}_{s, \mathcal{P}}$. Let $s \geq 2$, since $\varphi(s)=1$ we can define $R_{s}$ to be $R_{s}=-\mathbb{B}_{s, \mathcal{P}}^{2}+\sigma^{2} s^{2}\left(\widetilde{D}+\widetilde{A}_{P}^{0}\right)^{2}=-\nabla^{2}-s \Upsilon \nabla \sigma\left(\widetilde{D}+\widetilde{A}_{P}^{0}\right)-s \sigma\left(\widetilde{D}+\widetilde{A}_{P}^{0}\right) \Upsilon \nabla$. Now applying Duhamel's formula we get

$$
\begin{gathered}
\mathrm{e}^{-\mathbb{B}_{s, \mathcal{P}}^{2}}=\mathrm{e}^{-s^{2}\left(\widetilde{D}_{+}+\widetilde{A}_{P}^{0}\right)^{2}}+\int_{0}^{1} \mathrm{e}^{-u_{1} s^{2}\left(\widetilde{D}_{+} \widetilde{A}_{P}^{0}\right)^{2}} R_{s} \mathrm{e}^{-\left(1-u_{1}\right) s^{2}\left(\widetilde{D}_{+} \widetilde{A}_{P}^{0}\right)^{2}} d u_{1}+ \\
\int_{0}^{1} \int_{0}^{1-u_{1}} \mathrm{e}^{-u_{1} s^{2}\left(\widetilde{D}+\widetilde{A}_{P}^{0}\right)^{2}} R_{s} \mathrm{e}^{-u_{2} s^{2}\left(\widetilde{D}_{+} \widetilde{A}_{P}^{0}\right)^{2}} R_{s} \mathrm{e}^{-\left(1-u_{1}-u_{2}\right) s^{2}\left(\widetilde{D}_{+} \widetilde{A}_{P}^{0}\right)^{2}} d u_{2} d u_{1}+\ldots
\end{gathered}
$$

Let $m$ be a natural integer and $\mathcal{K}_{m}(s)$ be the projection of $\mathrm{e}^{-\mathbb{B}_{s, \mathcal{P}}^{2}}$ onto the space of operators with values in the set of homogeneous forms of degree $m$. We are going to show that there exists $\delta^{\prime}>0$ such that the $s$-family $\mathcal{K}_{m}(s)$ satisfies the decay property (DP) uniformly with respect to $\exp \left(-\delta^{\prime} s^{2}\right), s$ being in $\left[2,+\infty\left[\right.\right.$. Then, Proposition $3.4(3)$, with $\widetilde{A}=\sigma\left(\widetilde{D}+\widetilde{A}_{P}^{0}\right)$, will show that the same is true for the $s$-family $\widetilde{A} \circ \mathcal{K}_{m}(s)$. Hence Theorem 3.6 (1) will be an immediate consequence of Proposition 3.5.

So let us consider in the expansion (3.9) the integral over the $k$-simplex associated with $u_{1}, \ldots, u_{k}$. In this integral at least one of the following (non-negative) numbers:

$$
u_{1}, \ldots, u_{k}, 1-u_{1}-\cdots-u_{k}
$$


will be $\geq \frac{1}{k+1}, u_{j}$ for example. Then Lemma 3.7 (1) shows that the $s$-family of operators $\mathrm{e}^{-u_{j} s^{2}\left(\widetilde{D}+\widetilde{A}_{P}^{0}\right)^{2}}$ satisfies the decay property (DP) with respect to $\exp \left(-s^{2} \frac{\delta}{k+1}\right)$ as $s \geq 2$. Proposition 3.4 and Lemma 3.7 (2) show that this property will be preserved if we compose $\mathrm{e}^{-u_{j} s^{2}\left(\widetilde{D}_{+} \widetilde{A}_{P}^{0}\right)^{2}}$ on the right and on the left by the operators appearing in expansion (3.9). Part (1) of Theorem 3.6 is thus proved.

We now pass to part (2). So, a spectral section $\mathcal{P}$ being fixed, we have to show that the higher eta-invariant given by the integral (3.8) does not depend on $\varphi$ or on the choice of the $\mathcal{P}$-trivializing operator $A_{\mathcal{P}}^{0}$. So let $A_{\mathcal{P}}^{\prime 0}$ be another smoothing $\mathcal{P}$-trivializing operator as in Proposition 2.10, we consider a smooth homotopy of $\mathcal{P}$-trivializing operators $A_{\mathcal{P}}^{0}(r)$ defined for $r \in[0,1]$ such that $A_{\mathcal{P}}^{0}(0)=A_{\mathcal{P}}^{0}$ and $A_{\mathcal{P}}^{0}(1)=A_{\mathcal{P}}^{\prime 0}$. An explicit example is given by

$$
\mathcal{A}_{\mathcal{P}}^{0}(r)=(1-r) \mathcal{A}_{\mathcal{P}}^{0}+r \mathcal{A}_{\mathcal{P}}^{\prime 0}, \quad r \in[0,1],
$$

as an easy argument based on (3) of Proposition 2.10 shows (see [W1, Lemma 3.1]). For any $r \in[0,1]$, we consider the following superconnection:

$$
\mathbb{B}_{s, \mathcal{P}}(r)=\Upsilon \nabla+\sigma s\left(\widetilde{D}+\varphi(s) \widetilde{A}_{P}^{0}(r)\right),
$$

and the differential form in $\widehat{\Omega}_{*}\left(\mathcal{B}^{\infty}\right)$,

$$
\widehat{\eta}_{\mathcal{P}, r}(s)=\frac{2}{\sqrt{\pi}} \operatorname{STR}_{\mathrm{Cl}(1)}\left[\frac{d \mathbb{B}_{s, \mathcal{P}}(r)}{d s} \exp \left(-\left(\mathbb{B}_{s, \mathcal{P}}(r)^{2}\right)\right] .\right.
$$

Proceeding exactly as in the proof of Proposition 14 of [MPi2], we can show the following variation formula for the higher eta invariant:

Proposition 3.9. The differential forms $\widehat{\eta}_{\mathcal{P}, r}(s)$ depend smoothly on $r$ and we have:

$$
\frac{d}{d r} \widehat{\eta}_{\mathcal{P}, r}(s)=\frac{2}{\sqrt{\pi}} \frac{d}{d s}\left[s \operatorname{STR}_{\mathrm{Cl}(1)}\left(\sigma \varphi(s) \frac{d \widetilde{A}_{P}^{0}(r)}{d r} \exp \left(-\left(\mathbb{B}_{s, \mathcal{P}}(r)^{2}\right)\right)\right]\right.
$$

modulo exact forms and graded commutators.

We recall that $\varphi(s)=0$ for any $s \in[0,1]$. Moreover, the arguments used in the previous proof of the convergence of the integral of Theorem 3.6 (1) shows that

$$
\forall r \in[0,1], \quad \lim _{s \mapsto+\infty} s \operatorname{STR}_{\mathrm{Cl}(1)}\left(\sigma \varphi(s) \frac{d \widetilde{A}_{P}^{0}(r)}{d r} \exp \left(-\left(\mathbb{B}_{s, \mathcal{P}}(r)^{2}\right)\right)\right)=0 .
$$


Therefore, if we integrate the two members of equation (3.10) for $t \in[0,1]$ and $s \in[0,+\infty[$ we see that

$$
\int_{0}^{+\infty} \widehat{\eta}_{\mathcal{P}, 0}(s) d s=\int_{0}^{+\infty} \widehat{\eta}_{\mathcal{P}, 1}(s) d s
$$

modulo exact forms and graded commutators. Theorem 3.6 is therefore completely proved.

\section{Proof of the Technical Lemmas}

First we prove Lemma 3.7 (2). To this end we begin by recalling two standard estimates for the heat kernel $\exp \left(-s^{2} \widetilde{\not D}^{2}\right)$.

Let $\varepsilon>0$ be very small compared to the radius of injectivity of $\widetilde{N}$. We set, for any $(x, y) \in \widetilde{N}^{2}, R(x, y)=\max (d(x, y)-\varepsilon, 0)$. In [Lo2, p. 215], it is recalled that, for any $a \in \mathbb{N}$ and any $s \in] 0,1]$,

$$
\begin{gathered}
\forall x, y \in \tilde{N} \text { with } d(x, y)>2 \varepsilon \\
\left|\left(\widetilde{D D}^{a} \exp \left(-s^{2} \widetilde{D D}^{2}\right)\right)(x, y)\right| \leq C(a) \exp \left(-\frac{R^{2}(x, y)}{10 s^{2}}\right) .
\end{gathered}
$$

Next, since the heat kernel is $\Gamma$-invariant and almost Euclidean, the following asymptotic expansion is valid for $d(x, y) \leq 2 \varepsilon$ and $0<s<1$ :

$$
\exp \left(-s^{2} \widetilde{\not D}^{2}\right)(x, y) \sim\left(4 \pi s^{2}\right)^{-n / 2} \exp \left(-\frac{d^{2}(x, y)}{4 s^{2}}\right) \sum_{k \geq 0} s^{2 k} a_{k}(x, y),
$$

which can be differentiated at any order (in (4.2) $n$ is the dimension of $\widetilde{N}$ ). In both these estimates $x$ and $y$ are assumed to belong to the same connected component of $\widetilde{N}$ (see the remark after $(2.3)$ ).

Lemma 3.7 (2) is thus an easy consequence of the two estimates (4.1), (4.2) and of the following lemma.

Lemma 4.1. The operators $\mathrm{e}^{-s^{2}\left(\widetilde{D}+\widetilde{A}_{P}^{0}\right)^{2}}-\mathrm{e}^{-s^{2} \widetilde{D D}^{2}}$ satisfy estimates $(2.3)$ uniformly with respect to $s \in] 0,1]$. By definition, we will say that these operators are uniformly smoothing with respect to $s \in] 0,1]$.

Proof. We will give only the structure of the proof since the complete details of a similar argument are given in the proof of Proposition 1.6 of [LPi1]. We denote by $R$ the following smoothing operator: $R=\widetilde{D}_{\circ} \widetilde{A}_{P}^{0}+\widetilde{A}_{P}^{0} \circ \widetilde{D}+\left(\widetilde{A}_{P}^{0}\right)^{2}$. Applying Duhamel's formula we get

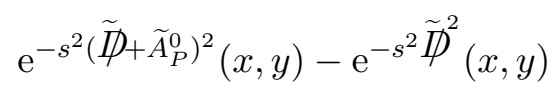




$$
\begin{gathered}
=\sum_{k \geq 1}\left(-s^{2}\right)^{k} \int_{0}^{1} \int_{0}^{1-u_{1}} \cdots \int_{0}^{1-u_{1} \cdots-u_{k-1}} \mathrm{e}^{-u_{1} s^{2} \widetilde{D D}^{2}} \operatorname{Re}^{-u_{2} s^{2} \widetilde{D D}^{2}} R \\
\cdots \operatorname{Re}^{-\left(1-u_{1} \cdots-u_{k}\right) s^{2} \widetilde{D D}^{2}}(x, y) d u_{1} \cdots d u_{k} .
\end{gathered}
$$

Let $(x, y) \in \tilde{N}^{2}$ be such that $d(x, y)>100$. We are going to show that the

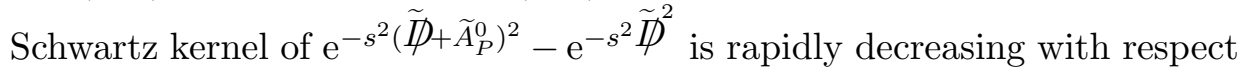
to $d(x, y)$ uniformly for $s \in] 0,1]$.

First we recall that the volume of the $k$-simplex appearing in (4.3) is $\frac{1}{k !}$. The expansion (4.3) is the sum of two terms corresponding to $\sum_{k \leq \sqrt{d(x, y)}}$ and $\sum_{k>\sqrt{d(x, y)}}$ respectively. We first observe that the term corresponding to $\sum_{k \leq \sqrt{d(x, y)}}$ is the sum of $[\sqrt{d(x, y)}]+1$ operators, each of them being the composition of at most $2 k \leq 2 \sqrt{d(x, y)}$ smoothing operators. We then check easily that the term corresponding to $\sum_{k \leq \sqrt{d(x, y)}}$ is of rapid decay with respect to $d(x, y)$ by using the two estimates (4.1) and (4.2).

Moreover, we check easily that the term corresponding to $\sum_{k>\sqrt{d(x, y)}}$ is also of rapid decay with respect to $d(x, y)$ by observing that for any $C>1, \sum_{k>\sqrt{d(x, y)}} \frac{C^{k}}{k !}$ is of rapid decay as $d(x, y) \mapsto+\infty$. Lemma 4.1 is thus proven.

Now, our goal is to show Lemma 3.7 (1). According to Proposition 2.10 $(2)$, we can find $\delta>0$ so that

$$
[-4 \delta, 4 \delta] \cap \operatorname{Spec}_{L^{2}}\left(\widetilde{D}+\widetilde{A}_{P}^{0}\right)^{2}=\emptyset .
$$

We consider the contour $\mathcal{C}$ in the complex plane defined by $\mathcal{C}=\{2 \delta+|\vartheta|+$ $i \vartheta / \vartheta \in \mathbb{R}\}$. Since $\left(\widetilde{D}+\widetilde{A}_{P}^{0}\right)^{2}$ is self-adjoint (4.4) implies that for any $\lambda \in \mathcal{C}$ the operator $\left(\widetilde{D}+\widetilde{A}_{P}^{0}\right)^{2}-\lambda \mathrm{Id}$ is invertible. Moreover we check easily that there exists $M>0$ such that

$$
\forall \lambda \in \mathcal{C}, \quad\left\|\left(\left(\widetilde{D}+\widetilde{A}_{P}^{0}\right)^{2}-\lambda \mathrm{Id}\right)^{-1}\right\|_{L^{2}(\widetilde{N}, \widetilde{E}) \rightarrow L^{2}(\widetilde{N}, \widetilde{E})} \leq \frac{M}{1+|\lambda|} .
$$

Using elementary spectral theory, we can write

$$
\mathrm{e}^{-s^{2}\left(\widetilde{D}_{+}+\widetilde{A}_{P}^{0}\right)^{2}}=\frac{i}{2 \pi} \int_{\mathcal{C}} \mathrm{e}^{-s^{2} \lambda}\left(\left(\widetilde{D}+\widetilde{A}_{P}^{0}\right)^{2}-\lambda \mathrm{Id}\right)^{-1} d \lambda
$$

We begin by proving the estimates of Lemma $3.7(1)$ when $(x, y)$ remains in an $\varepsilon$-neighborhood of the diagonal, i.e. $d(x, y)<\varepsilon$. Since the Schwartz kernel of $\mathrm{e}^{-s^{2}\left(\widetilde{D}_{+}+\widetilde{A}_{P}^{0}\right)^{2}}$ is $\Gamma$-invariant, we can assume that $(x, y)$ remains in 
a compact subset of $\widetilde{N} \times \widetilde{N}$. Using the spectral representation (4.6) and estimate (4.5) we see that for any $N \in \mathbb{N}$ the $L^{2}$-operator norm of

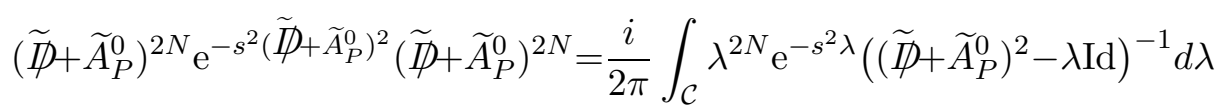

is bounded by $C_{N} \mathrm{e}^{-s^{2} \delta}$. So the distribution order of the successive derivatives of the distribution Schwartz kernel of $\mathrm{e}^{-s^{2}\left(\widetilde{D}_{+}+\widetilde{A}_{P}^{0}\right)^{2}}$ remain fixed. Therefore, using Sobolev's injection theorem, we get immediately the estimates Lemma 3.7 (1) for $d(x, y)<\varepsilon$. So, using the representation (4.6), we see that the rest of Lemma 3.7 (1) is a consequence of the following:

Lemma 4.2. (1) For any $\lambda \in \mathcal{C}$ the Schwartz kernel of $\left(\left(\widetilde{D}+\widetilde{A}_{P}^{0}\right)^{2}-\lambda \mathrm{Id}\right)^{-1}$ is smooth outside the diagonal and of rapid decay as $d(x, y) \mapsto+\infty$.

(2) Let $\varepsilon$ be a positive real. Then for each $(a, q)$ in $\mathbb{N} \times \mathbb{N}$ we can find $C_{q, a}>0$ such that for any $\lambda \in \mathcal{C}$

$$
\sup _{(x, y) \in \widetilde{N}^{2}, d(x, y)>\varepsilon}\left|\left(\widetilde{D}+\widetilde{A}_{P}^{0}\right)^{2 a}\left(\left(\widetilde{D}+\widetilde{A}_{P}^{0}\right)^{2}-\lambda \mathrm{Id}\right)^{-1}(x, y)\right|(1+d(x, y))^{q} \leq C_{q, a} .
$$

Proof. Let us prove (1). Let $\lambda \in \mathcal{C}$. Let $\varepsilon^{\prime}$ be a small positive real. We can certainly find an $\varepsilon^{\prime}$-local parametrix $G_{\lambda} \in \Psi^{-2}(\widetilde{N}, \widetilde{E})$ of $\left(\widetilde{D}+\widetilde{A}_{P}^{0}\right)^{2}-\lambda \mathrm{Id}$,

$$
\left[\left(\widetilde{D}+\widetilde{A}_{P}^{0}\right)^{2}-\lambda \mathrm{Id}\right] \circ G_{\lambda}=\mathrm{Id}-T_{\lambda},
$$

where $T_{\lambda}$ is a $\Gamma$-invariant smoothing operator satisfying (2.3) (i.e. rapidly decreasing).

Moreover, using the assertion (4.4) and the definition of $\mathcal{C}$, one checks easily that $\left(\widetilde{D}+\widetilde{A}_{P}^{0}\right)^{2}-\lambda$ Id induces, in the $\Lambda$-pseudodifferential calculus of Mishenko-Fomenko ([MiF]) on $N$, an invertible operator denoted by $\left(\not D+\mathcal{A}_{P}^{0}\right)^{2}-\lambda \mathrm{Id}$.

Therefore Proposition 1.6 of [LPi1] allows us to see that $\left(\left(\widetilde{D}+\widetilde{A}_{P}^{0}\right)^{2}-\right.$ $\lambda \mathrm{Id})^{-1}$ sends continuously the Schwartz space $S(\widetilde{N}, \widetilde{E})$ into itself. It is then clear that the Schwartz kernel of

$$
\left(\left(\widetilde{D}+\widetilde{A}_{P}^{0}\right)^{2}-\lambda \mathrm{Id}\right)^{-1}=G_{\lambda}+\left(\left(\widetilde{D}+\widetilde{A}_{P}^{0}\right)^{2}+\lambda \mathrm{Id}\right)^{-1} \circ T_{\lambda}
$$

is smooth and of rapid decay outside the diagonal. Moreover we easily get the (uniform) estimates of (2) if $\lambda$ remains in the compact subset $\mathcal{C} \cap$ $\{\lambda, / \Re \lambda \leq 1\}$. Now we are going to prove (2) when $\lambda$ belongs to $\mathcal{C}_{1}=\mathcal{C} \cap$ $\{\lambda, / \Re \lambda \geq 1\}$. Mixing the definitions of $[\mathrm{MiF}]$ and $[\mathrm{G}$, p. 50], we define a $\Lambda$ pseudodifferential calculus $\Psi_{\Lambda, \lambda}^{*}(N, \mathcal{E})$ with parameter $\lambda \in \mathcal{C}_{1}$. An operator belonging to $\Psi_{\Lambda, \lambda}^{m}(N, \mathcal{E})$ is defined in each suitably small local chart $U(\subset N)$ by a classical symbol $\sigma(x, \xi, \lambda)$ belonging to $C^{\infty}\left(T^{*} U \times \mathcal{C}_{1} ; M_{\operatorname{rank} E}(\Lambda)\right)$, 
$M_{\operatorname{rank} E}(\Lambda)$ denoting the matrix algebra on $\Lambda$ and satisfying the following estimates:

$\forall(x, \xi, \lambda) \in T^{*} U \times \mathcal{C}_{1},\left\|\partial_{x}^{\alpha} \partial_{\xi}^{\beta} \partial_{\lambda}^{a} \sigma(x ; \xi, \lambda)\right\| \leq C_{\alpha, \beta, a}\left(1+|\xi|+|\lambda|^{1 / 2}\right)^{m-|\beta|-2 a}$.

Moreover, we assume that $\sigma(x ; \xi, \lambda)$ admits an asymptotic expansion $\sim \sum_{k \in \mathbb{N}} \sigma_{m-k}(x, \xi, \lambda)$ where the symbols $\sigma_{m-k}(x, \xi, \lambda)$ are homogeneous of degree $m-k$, more precisely this means that for any real $t \geq 1$

$$
\sigma_{m-k}\left(x, t \xi, t^{2} \lambda\right)=t^{m-k} \sigma_{m-k}(x, \xi, \lambda)
$$

In a similar way, one defines a $\mathcal{B}^{\infty}$-pseudo-differential calculus with parameter $\Psi_{\mathcal{B}^{\infty}, \lambda}^{m}\left(N, \mathcal{E}^{\infty}\right)$, we simply replace the $C^{*}$-norm in $(4.7)$ by the semi-norms induced by those of $\mathcal{B}^{\infty}$.

Clearly, $\not^{2}-\lambda \mathrm{Id}$ is elliptic and admits a parametrix $\mathcal{G}_{\lambda} \in \Psi_{\mathcal{B}^{\infty}, \lambda}^{-2}\left(N, \mathcal{E}^{\infty}\right)$ such that its associated operator $G_{\lambda}$ on the covering (cf. equation (3.4)) is $\varepsilon^{\prime}$-local. So there exist $\mathcal{T}_{\lambda}^{L}, \mathcal{T}_{\lambda}^{R} \in \Psi_{\mathcal{B} \infty, \lambda}^{-\infty}\left(N, \mathcal{E}^{\infty}\right)$ such that

$$
\left(\not D^{2}-\lambda \mathrm{Id}\right) \circ \mathcal{G}_{\lambda}=\mathrm{Id}-\mathcal{T}_{\lambda}^{R}, \quad \mathcal{G}_{\lambda} \circ\left(\not{D}^{2}-\lambda \mathrm{Id}\right)=\mathrm{Id}-\mathcal{T}_{\lambda}^{L} .
$$

Moreover since $\mathcal{G}_{\lambda}$ is of negative order, the two $\lambda$-families of operators $\mathcal{T}_{\lambda}^{1}, \mathcal{T}_{\lambda}^{2}$ defined by

$$
\begin{aligned}
& \mathcal{T}_{\lambda}^{1}=\mathcal{T}_{\lambda}^{R}-\left[\not \mathcal{D} \circ \mathcal{A}_{P}^{0}+\mathcal{A}_{P}^{0} \circ \not{D}+\left(\mathcal{A}_{P}^{0}\right)^{2}\right] \circ \mathcal{G}_{\lambda} \\
& \mathcal{T}_{\lambda}^{2}=\mathcal{T}_{\lambda}^{L}-\mathcal{G}_{\lambda} \circ\left[\not \mathcal{D} \circ \mathcal{A}_{P}^{0}+\mathcal{A}_{P}^{0} \circ \not{D}+\left(\mathcal{A}_{P}^{0}\right)^{2}\right]
\end{aligned}
$$

will be uniformly bounded in $\Psi_{\mathcal{B}^{\infty}}^{-\infty}\left(N, \mathcal{E}^{\infty}\right)$ for $\lambda \in \mathcal{C}_{1}$.

Therefore, we can write for any $\lambda \in \mathcal{C}_{1}$

$$
\left(\left(\not \mathcal{D}+\mathcal{A}_{P}^{0}\right)^{2}-\lambda \mathrm{Id}\right) \circ \mathcal{G}_{\lambda}=\mathrm{Id}-\mathcal{T}_{\lambda}^{1}, \quad \mathcal{G}_{\lambda} \circ\left(\left(\not \mathcal{D}+\mathcal{A}_{P}^{0}\right)^{2}-\lambda \mathrm{Id}\right)=\mathrm{Id}-\mathcal{T}_{\lambda}^{2} .
$$

Applying $\left(\left(\not \mathcal{D}+\mathcal{A}_{P}^{0}\right)^{2}-\lambda \mathrm{Id}\right)^{-1}$ to the left and right sides of the two equalities above, we get

$$
\left(\left(\not \mathcal{D}+\mathcal{A}_{P}^{0}\right)^{2}-\lambda \mathrm{Id}\right)^{-1}=\mathcal{G}_{\lambda}+\mathcal{T}_{\lambda}^{2} \circ \mathcal{G}_{\lambda}+\mathcal{T}_{\lambda}^{2} \circ\left(\left(\not \mathcal{D}+\mathcal{A}_{P}^{0}\right)^{2}-\lambda \mathrm{Id}\right)^{-1} \circ \mathcal{T}_{\lambda}^{1} .
$$

Considering the associated operators on the covering $\tilde{N}$, the previous identity becomes

$$
\left(\left(\widetilde{D}+\widetilde{A}_{P}^{0}\right)^{2}-\lambda \mathrm{Id}\right)^{-1}=G_{\lambda}+T_{\lambda}^{2} \circ G_{\lambda}+T_{\lambda}^{2} \circ\left(\left(\widetilde{D}+\widetilde{A}_{P}^{0}\right)^{2}-\lambda \mathrm{Id}\right)^{-1} \circ T_{\lambda}^{1},
$$

where $T_{\lambda}^{1}, T_{\lambda}^{2}$ are uniformly bounded families of rapidly decreasing smoothing operators. We observe that the two family of operators $G_{\lambda}, T_{\lambda}^{2} \circ G_{\lambda}$ satisfy the required estimates of Lemma $4.2(2)$, so in order to complete the proof of the lemma we just have to show that the operators $T_{\lambda}^{2} \circ\left(\left(\widetilde{D}+\widetilde{A}_{P}^{0}\right)^{2}-\right.$ $\lambda \mathrm{Id})^{-1}$ 
$\circ T_{\lambda}^{1}$ are rapidly decreasing smoothing on the covering uniformly with respect to $\lambda \in \mathcal{C}_{1}$. To show this it is enough to prove that $\left(\left(\widetilde{D}+\widetilde{A}_{P}^{0}\right)^{2}-\lambda \mathrm{Id}\right)^{-1}$ sends uniformly $S(\widetilde{N}, \widetilde{E})$ into itself with respect to $\lambda \in \mathcal{C}_{1}$. Since the $L^{2}(N, \mathcal{E})$-operator norm of $\left(\left(\not D+\mathcal{A}_{P}^{0}\right)^{2}-\lambda \mathrm{Id}\right)^{-1}$ goes to zero as $\lambda \in \mathcal{C}_{1}$ goes to infinity, we see that the $\lambda$-family $\left(\left(\not \mathcal{D}+\mathcal{A}_{P}^{0}\right)^{2}-\lambda \mathrm{Id}\right)^{-1}$ is uniformly bounded in $\Psi_{\Lambda}^{-2}(N, \mathcal{E})$ for $\lambda \in \mathcal{C}_{1}$. Hence using equation (4.8), we can certainly find a family of operators $\mathcal{V}_{\lambda} \in \Psi_{\mathcal{B} \infty}^{-2}\left(N, \mathcal{E}^{\infty}\right)$ uniformly bounded for $\lambda \in \mathcal{C}_{1}$ such that $\left(\left(\not{D}+\mathcal{A}_{P}^{0}\right)^{2}-\lambda \mathrm{Id}\right)^{-1}-\mathcal{V}_{\lambda}$ is "small enough" in the sense that for any $\lambda \in \mathcal{C}_{1}$ the $L^{2}(N, \mathcal{E})$-operator norm of $-\mathrm{Id}+\left(\left(\not \mathcal{D}+\mathcal{A}_{P}^{0}\right)^{2}-\lambda \mathrm{Id}\right) \circ \mathcal{V}_{\lambda}$ is $<1 / 2$. Let $\widetilde{D}, \widetilde{A}_{P}^{0}, V_{\lambda}$ be the operators on the covering $\widetilde{N}$ associated with $\not{D}, \mathcal{A}_{P}^{0}, \mathcal{V}_{\lambda}$. The previous uniform estimate of the $L^{2}(N, \mathcal{E})$-operator norms allows us to apply Proposition 1.6 of [LPi1] so as to see that

$$
\left(\operatorname{Id}-\operatorname{Id}+\left(\left(\widetilde{D}+\widetilde{A}_{P}^{0}\right)^{2}-\lambda \mathrm{Id}\right) \circ V_{\lambda}\right)^{-1}
$$

sends continuously $S(\widetilde{N}, \widetilde{E})$ into itself uniformly with respect to $\lambda \in \mathcal{C}_{1}$. Applying $V_{\lambda}$ to the left of (4.10) we see that $\left(\left(\widetilde{D}+\widetilde{A}_{P}^{0}\right)^{2}-\lambda \mathrm{Id}\right)^{-1}$ sends uniformly $S(\widetilde{N}, \widetilde{E})$ into itself with respect to $\lambda \in \mathcal{C}_{1}$. Lemma 4.2 is therefore proved.

\section{Variation Properties of $\mathcal{P}$-higher Eta Invariants and the Noncommutative Spectral Flow}

In all this section we shall assume the group $\Gamma$ to be virtually nilpotent. Let $\not D: C^{\infty}\left(N, \mathcal{F}^{\infty}\right) \rightarrow C^{\infty}\left(N, \mathcal{F}^{\infty}\right)$ be a Dirac operator as in sect. 2 and let us assume $\operatorname{Ind}(\not \mathcal{D})=0$ in $K_{1}\left(C_{r}^{*}(\Gamma)\right)$; thus there exists an infinite number of spectral sections.

First of all we investigate the dependence of the $\mathcal{P}$-higher eta invariant on the choice of the spectral section $\mathcal{P}$. Given a second spectral section $\mathcal{Q}$ it is possible to define a difference class in $K^{0}\left(\mathcal{B}^{\infty}\right) \equiv K^{0}(\Lambda)$ (see [W1]). We have

Theorem 5.1. Let $\mathcal{Q} \in \Psi_{\mathcal{B}^{\infty}}^{0}\left(N, \mathcal{F}^{\infty}\right)$ be a different spectral section for $\not{D}$. Then

$$
\widehat{\eta}_{\mathcal{P}}-\widehat{\eta}_{\mathcal{Q}}=2 \operatorname{Ch}[\mathcal{P}-\mathcal{Q}] \text { in } \bar{H}_{*}\left(\mathcal{B}^{\infty}\right) .
$$

Proof. The proof makes use of the Berline-Vergne diagonalization lemma on Galois covering as presented in [LPi1]. It follows the same lines as the proof of Proposition 17 of [MPi2] and Theorem (3.2) of [LPi1]. Notice that part (2) of Corollary 2.9 allows us to choose the two auxiliary spectral sections of [MPi2] in the $\mathcal{B}^{\infty}$-calculus. We leave the details to the interested reader. 
Next we connect the variation of the higher eta invariant with the noncommutative spectral flow of $\mathrm{Wu}$ (see [DZ] for the commutative case).

Thus let $\not{D}_{u}: C^{\infty}\left(N, \mathcal{F}^{\infty}\right) \rightarrow C^{\infty}\left(N, \mathcal{F}^{\infty}\right), u \in[0,1]$, be a smooth 1-parameter family of Dirac operators as above (thus each $\mathbb{D}_{u}$ has vanishing $K_{1}$-index). We denote this family by $\left\{\mathcal{P}_{u}\right\}$. Since $\operatorname{Ind}\left(\left\{\mathscr{D}_{u}\right\}\right)=0$ in $K_{1}\left(C[0,1] \otimes \mathcal{B}^{\infty}\right)$ it follows that there exists a noncommutative spectral section $\mathcal{P}=\left\{\mathcal{P}_{u}\right\}$ associated to $\left\{\not{D}_{u}\right\}$. According to Theorem 2.6 (2) we can always choose $\mathcal{P}_{u} \in \Psi_{\mathcal{B}^{\infty}}^{0}\left(N, \mathcal{F}^{\infty}\right) \forall u$. If $\mathcal{Q}_{0}, \mathcal{Q}_{1}$ are spectral sections associated to $\not_{0}, \mathbb{D}_{1}$ then $\mathrm{Wu}$ has defined the noncommutative spectral flow $\operatorname{sf}\left(\left\{\not{D}_{u}\right\} ; \mathcal{Q}_{0}, \mathcal{Q}_{1}\right)$ from $\left(\mathcal{D}_{0}, \mathcal{Q}_{0}\right)$ to $\left(\mathcal{D}_{1}, \mathcal{Q}_{1}\right)$ through $\left\{\mathcal{D}_{u}\right\}$ as the $K_{0}\left(\mathcal{B}^{\infty}\right)$ class

$$
\operatorname{sf}\left(\left\{\mathcal{P}_{u}\right\} ; \mathcal{Q}_{0}, \mathcal{Q}_{1}\right):=\left[\mathcal{Q}_{1}-\mathcal{P}_{1}\right]-\left[\mathcal{Q}_{0}-\mathcal{P}_{0}\right] \in K_{0}\left(\mathcal{B}^{\infty}\right) .
$$

The notation is meaningful since the definition does not depend on the particular choice of the (total) spectral section $\mathcal{P}=\left\{\mathcal{P}_{u}\right\}$.

Let us fix a smooth 1-parameter family $\left\{\mathcal{A}_{u}^{0}\right\}$ with the property that $\mathcal{A}_{u}^{0}$ is a $\mathcal{P}_{u}$-trivializing operator for $\mathcal{P}_{u}$ (see (4) of Proposition 2.10). For each $u \in[0,1]$ we define a rescaled superconnection by

$$
\mathbb{B}(s, u)=\Upsilon \nabla+\sigma s\left(\mathcal{D}_{u}+\varphi(s) \mathcal{A}_{u}^{0}\right) .
$$

Here $\varphi$ is the cut-off function of (3.1). We will denote (for simplicity) by $\widehat{\eta}(s, u)$ the higher eta-integrand,

Thus

$$
\widehat{\eta}(s, u)=\frac{2}{\pi} \operatorname{STR}_{\mathrm{Cl}(1)}\left[\frac{d \mathbb{B}(s, u)}{d s} \exp \left(-\mathbb{B}(s, u)^{2}\right)\right] .
$$

$$
\widehat{\eta}_{\mathcal{P}_{u}}\left(\mathcal{D}_{u}\right)=\int_{0}^{+\infty} \widehat{\eta}(s, u) d s .
$$

In order to avoid the double subscript we shall also employ the notation

$$
\widehat{\eta}_{\mathcal{P}_{u}}\left(\mathcal{D}_{u}\right)=\widehat{\eta}\left(\mathcal{D}_{u}, \mathcal{P}_{u}\right) \text {. }
$$

We define $\dot{\mathcal{D}}_{u}$ to be the operator obtained by differentiating $\mathscr{D}_{u}$ with respect to $u$. Then, we observe that as $s$ goes to $0^{+}$,

$$
-\frac{2}{\pi} \operatorname{STR}_{\mathrm{Cl}(1)}\left(s \sigma \dot{\mathcal{D}}_{u} \exp \left(-\mathbb{B}(s, u)^{2}\right)\right)
$$

admits an asymptotic expansion with respect to the integral powers of $s$. We will denote by $a_{0}(u) \in \widehat{\widehat{\Omega}}_{*}\left(\mathcal{B}^{\infty}\right)$ the coefficient of $s^{0}$. Proceeding as in sect. 3 it is easy to obtain the following:

Proposition 5.2. For each $u \in[0,1]$ we have

$$
\frac{\partial}{\partial u} \widehat{\eta}\left(\mathcal{D}_{u}, \mathcal{P}_{u}\right)=a_{0}(u) \text { in } \overline{\widehat{\Omega}}_{*}\left(\mathcal{B}^{\infty}\right) / d \widehat{\Omega}_{*}\left(\mathcal{B}^{\infty}\right)
$$


Hence,

$$
\widehat{\eta}\left(\mathcal{D}_{1}, \mathcal{P}_{1}\right)-\widehat{\eta}\left(\mathcal{D}_{0}, \mathcal{P}_{0}\right)=\int_{0}^{1} a_{0}(u) d u
$$

Using the previous proposition and Theorem 5.1 we obtain immediately, as in [DZ, Theorem 2.12], the main result of this section.

Theorem 5.3. Let $\left\{\not_{u}\right\}$ be a smooth 1-parameter family of Dirac operators and $\mathcal{Q}_{0}, \mathcal{Q}_{1}$ be noncommutative spectral sections for $\mathbb{D}_{0}, \mathbb{D}_{1}$ respectively. Then the following formula holds

$2 \mathrm{Ch}\left(\operatorname{sf}\left(\left\{\mathcal{D}_{u}\right\} ; \mathcal{Q}_{0}, \mathcal{Q}_{1}\right)\right)=\widehat{\eta}\left(\mathcal{D}_{1}, \mathcal{Q}_{1}\right)-\widehat{\eta}\left(\mathcal{D}_{0}, \mathcal{Q}_{0}\right)-\int_{0}^{1} a_{0}(u) d u$ in $\bar{H}_{*}\left(\mathcal{B}^{\infty}\right)$.

Proof. By definition we have

$$
2 \operatorname{Ch}\left(\operatorname{sf}\left(\left\{\not_{u}\right\} ; \mathcal{Q}_{0}, \mathcal{Q}_{1}\right)\right)=2 \operatorname{Ch}\left[\mathcal{P}_{0}-\mathcal{Q}_{0}\right]-2 \operatorname{Ch}\left[\mathcal{P}_{1}-\mathcal{Q}_{1}\right]
$$

Moreover, according to Theorem 5.1,

$$
\begin{aligned}
& \widehat{\eta}\left(\mathcal{D}_{0}, \mathcal{P}_{0}\right)-\widehat{\eta}\left(\mathcal{D}_{0}, \mathcal{Q}_{0}\right)=2 \operatorname{Ch}\left[\mathcal{Q}_{0}-\mathcal{P}_{0}\right] \\
& \widehat{\eta}\left(\mathcal{D}_{1}, \mathcal{P}_{1}\right)-\widehat{\eta}\left(\mathcal{D}_{1}, \mathcal{Q}_{1}\right)=2 \operatorname{Ch}\left[\mathcal{Q}_{1}-\mathcal{P}_{1}\right]
\end{aligned}
$$

Using the three equations (5.4), (5.5), (5.6) we get Theorem 5.3 at once.

\section{Higher Atiyah-Patodi-Singer Index Classes}

In order to orient the reader we first recall the family (i.e. commutative) case of what follows (see [MPi2]). Let $\not D=\left(D_{z}\right)_{z \in B}$ be a Dirac family on even dimensional manifolds with boundary. Proposition 2.10, when applied to the boundary Dirac family $\not_{0}$, gives a smooth family of smoothing operators $\widetilde{A}_{P}^{0}$ satisfying the analogues of (1), (2), (3) in Proposition 2.10. From $\widetilde{A}_{P}^{0}$ it is then possible to construct a $b$-pseudodifferential perturbation $\not D^{+}+A_{P}^{+}$of the original family $\not^{+}$, with $A_{P}^{+}$of order $(-\infty)$ in the $b$-calculus, satisfying two crucial properties. First, that $\not D^{+}+A_{P}^{+}$has invertible boundary family; this implies that $\not D^{+}+A_{P}^{+}$defines a smooth family of Fredholm operators acting on $L^{2}$. Second, that the two index classes, the one defined by generalized APS boundary conditions, $\operatorname{Ind}\left(\not D^{+}, \mathcal{P}\right)$, and the one defined by the $L^{2}$-Fredholm family $\not^{+}+A_{P}^{+}$are equal in $K^{0}(B) \equiv K_{0}\left(C^{0}(B)\right)$ : $\operatorname{Ind}\left(\not D^{+}, \mathcal{P}\right)=\operatorname{Ind}\left(\not \not^{+}+A_{P}^{+}\right)$. The family APS index theory of $[\mathrm{MPi} 2,3]$ is obtained by working on the latter class. One could very well define the index class associated to $\not D$ and $P$ as $\operatorname{Ind}\left(\not D^{+}+A_{P}^{+}\right)$, since it can be shown that the latter only depends on $I D$ and $P$ and not on the particular choices 
involved in the definition of the perturbation $A_{P}^{+}$. This is the route that we follow here in the noncommutative context.

Thus let $\Gamma \rightarrow \widetilde{M} \rightarrow M$ be an even dimensional Galois covering with boundary. For the time being we only assume that the discrete group $\Gamma$ is finitely presented. Let $\not D \in \operatorname{Diff}_{b}^{1}(M, E)$ be a $b$-Dirac operator and let $\widetilde{\not D} \in \operatorname{Diff}_{b, \Gamma}^{1}(\widetilde{M}, \widetilde{E})$ (resp. $\left.\not D \in \operatorname{Diff}_{b, \Lambda}^{1}(M, \mathcal{E})\right)$ be the associated operators on the covering $\widetilde{M}$ (resp. in the Mishenko-Fomenko $b$-calculus). According to Theorem 2.7 there always exists a spectral section $\mathcal{P} \in \Psi_{\Lambda}^{0}\left(\partial M, \mathcal{E}_{0}\right)$ associated to the boundary operator $\not{D}_{0} \in \operatorname{Diff}_{\Lambda}^{1}\left(\partial M, \mathcal{E}_{0}\right)$. We fix such a spectral section together with a $\mathcal{P}$-trivializing operator $\mathcal{A}_{\mathcal{P}}^{0} \in \Psi_{\Lambda}^{-\infty}\left(\partial M, \mathcal{E}_{0}\right)$ as in Proposition 2.10. In the next lemma we will adopt the identifications near the boundary explained in sect. 1. Furthermore we shall begin to make use of the $\Lambda$-Mishenko-Fomenko $b$-calculus. This is defined as in the commutative case, thus in terms of the Schwartz kernels on the $b$-stretched product $M_{b}^{2}$, but with complex functions replaced by functions with values in $\Lambda$. The fundamental result in the $\Lambda$-Mishenko-Fomenko $b$-calculus is the parametrix construction: if $\not{D} \in \operatorname{Diff}_{b, \Lambda}^{m}\left(M, \mathcal{E}^{+}, \mathcal{E}^{-}\right)$is $b$-elliptic and has invertible indicial family $I(\mathcal{D}, \lambda), \lambda \in \mathbb{R}$ then it admits an inverse $\mathcal{Q}$ modulo $\Lambda$-compact operators (with $\mathcal{Q}$ in the $b$-calculus with bounds $\Psi_{b, \Lambda}^{-m, \varepsilon}, \varepsilon>0$ ). More details can be found in [LPi1, Sect. 11].

Let $\rho \in C_{c}^{\infty}\left(\mathbb{R}, \mathbb{R}^{+}\right)$be a nonnegative, even smooth test function such that $\int_{\mathbb{R}} \rho(x) d x=1$. We then consider the Fourier-Transform of $\rho_{\varepsilon}$ defined as $\rho_{\varepsilon}(t)=\rho\left(\frac{t}{\varepsilon}\right) \varepsilon^{-1}$,

$$
\widehat{\rho}_{\varepsilon}(z)=\int_{\mathbb{R}} \mathrm{e}^{-i t z} \rho\left(\frac{t}{\varepsilon}\right) \varepsilon^{-1} d t
$$

and observe that $\widehat{\rho_{\varepsilon}}(0)=1$.

Lemma 6.1. There exists an operator $\mathcal{A}_{\mathcal{P}}^{+} \in \Psi_{b, \Lambda}^{-\infty}\left(M, \mathcal{E}^{+}, \mathcal{E}^{-}\right)$with the following properties:

(1) There exists $R \in \mathbb{R}^{+*}$, such that for each $z \in \mathbb{C}$ the indicial family $I\left(\mathcal{A}_{\mathcal{P}}^{+}, z\right)$ satisfies $\psi\left(\mathcal{D}_{0}\right) \circ I\left(\mathcal{A}_{\mathcal{P}}^{+}, z\right)=0 \quad \forall \psi \in C^{\infty}([-\infty, \infty])$ with $\psi(t)=0$ for $|t|<R$.

(2) For each $\lambda \in \mathbb{R}$ the indicial family of the operator $\not{D}_{\mathcal{P}}^{+} \equiv \not{D}^{+}+\mathcal{A}_{\mathcal{P}}^{+}$is invertible as an element of $\mathrm{B}_{\Lambda}\left(\mathcal{H}^{k}\left(\partial M, \mathcal{E}_{0}\right), \mathcal{H}^{k-1}\left(\partial M, \mathcal{E}_{0}\right)\right)$ for each $k \in \mathbb{Z}$.

Proof. The proof proceeds exactly as that of Lemma 9 of [MPi2]. By construction $\mathcal{A}_{\mathcal{P}}^{+}$is obtained by extending its indicial operator $I\left(\mathcal{A}_{\mathcal{P}}^{+}\right)$through 
a cut-off function $f$ equal to 1 over $\partial M$. In order to define the indicial operator it suffices to fix the associated indicial family. This is, by definition, $I\left(\mathcal{A}_{\mathcal{P}}^{+}, z\right)=\widehat{\rho}_{\varepsilon}(z) \mathcal{A}_{\mathcal{P}}^{0}$. By construction,

$$
I\left(\not{D}_{\mathcal{P}}^{+}, z\right)=\not{D}_{0}+i z+\widehat{\rho}_{\varepsilon}(z) \mathcal{A}_{\mathcal{P}}^{0}
$$

this is invertible for each $z \in \mathbb{R}$ since $\widehat{\rho}_{\varepsilon}(z)$ is real for $z \in \mathbb{R}$ and $\mathcal{A}_{\mathcal{P}}^{0}$ is self-adjoint. The lemma is proved.

Theorem 6.2. For any $k \in \mathbb{N}$, the operator $\not{D}^{+}+\mathcal{A}_{\mathcal{P}}^{+}$is $\Lambda$-Fredholm as an operator $\mathcal{H}_{b}^{k}\left(M, \mathcal{E}^{+}\right) \rightarrow \mathcal{H}_{b}^{k-1}\left(M, \mathcal{E}^{-}\right)$with index class $[\mathcal{L}(\mathcal{P})]-[\mathcal{N}(\mathcal{P})] \in$ $K_{0}(\Lambda) \equiv K_{0}\left(C_{r}^{*}(\Gamma)\right)$ independent of $k$.

Proof. It suffices to apply Lemma 6.1, the parametrix construction as explained above and Theorem 2.4 of [MiF]. That the index class is independent of $k$ follows also from the parametrix construction and more precisely from the regularity properties of the remainders.

Definition 6.3. The index class $\operatorname{Ind}\left(\not{D}^{+}, \mathcal{P}\right)$ associated to the Dirac operator $\mathscr{D}^{+} \in \operatorname{Diff}_{\Lambda}^{1}\left(M, \mathcal{E}^{+}, \mathcal{E}^{-}\right)$and the spectral section $\mathcal{P}$ for the boundary operator $\mathscr{D}_{0}$ is, by definition, the index class associated to $\not{D}^{+}+\mathcal{A}_{\mathcal{P}}^{+}$: $\operatorname{Ind}\left(\not{D}^{+}, \mathcal{P}\right) \equiv \operatorname{Ind}\left(\not{D}^{+}+\mathcal{A}_{\mathcal{P}}^{+}\right)=[\mathcal{L}(\mathcal{P})]-[\mathcal{N}(\mathcal{P})] \in K_{0}\left(C_{r}^{*}(\Gamma)\right)$.

Proposition 6.4. The class $\operatorname{Ind}\left(\not{D}^{+}, \mathcal{P}\right) \in K_{0}\left(C_{r}^{*}(\Gamma)\right)$ depends only on $\not D$ and the spectral section $\mathcal{P}$ and not on the particular choices involved in the definition of $\mathcal{A}_{\mathcal{P}}^{+}$.

Proof. The definition of $\mathcal{A}_{\mathcal{P}}^{+}$depends on the test function $\rho$, the parameter $\varepsilon$, the cut-off function $f$ and on the $\mathcal{P}$-trivializing operator $\mathcal{A}_{\mathcal{P}}^{0}$. Let $\mathcal{B}_{\mathcal{P}}^{+}$be a different perturbation, corresponding to different choices $\rho^{\prime}, \varepsilon^{\prime}, f^{\prime}, \mathcal{B}_{\mathcal{P}}^{0}$. It suffices to show that

$$
\not{D}^{+}+r \mathcal{A}_{\mathcal{P}}^{+}+(1-r) \mathcal{B}_{\mathcal{P}}^{+}, \quad r \in[0,1]
$$

is a homotopy through $\Lambda$-Fredholm operators. To see this point consider the indicial family associated to $(6.4)$; by (6.3) this is equal to

$$
\mathcal{D}_{0}+i \lambda+r \widehat{\rho_{\varepsilon}}(\lambda) \mathcal{A}_{\mathcal{P}}^{0}+(1-r) \widehat{\rho_{\varepsilon^{\prime}}^{\prime}}(\lambda) \mathcal{B}_{\mathcal{P}}^{0} .
$$

By the $b$-calculus, it is enough to prove that this operator is invertible for each $r \in[0,1]$ and for each $\lambda \in \mathbb{R}$. Since $\widehat{\rho_{\varepsilon}}(\lambda), \widehat{\rho_{\varepsilon^{\prime}}^{\prime}}(\lambda)$ are real $\forall \lambda \in \mathbb{R}$ and $\mathcal{A}_{\mathcal{P}}^{0}, \mathcal{B}_{\mathcal{P}}^{0}$ are self-adjoint we are reduced to check the statement at $\lambda=0$ where it follows from Proposition 2.10 by writing

$$
\not{D}_{0}+r \mathcal{A}_{\mathcal{P}}^{0}+(1-r) \mathcal{B}_{\mathcal{P}}^{0}=\left(r\left|\mathcal{D}_{0}+\mathcal{A}_{\mathcal{P}}^{0}\right|+(1-r)\left|\mathcal{D}_{0}+\mathcal{B}_{\mathcal{P}}^{0}\right|\right)(2 \mathcal{P}-\mathrm{Id}) .
$$

The proposition is proved. 
REMARK. A different method for defining the index class associated to $\not{D}^{+}$and $\mathcal{P}$, more in the spirit of the original APS work, has been recently given by $\mathrm{Wu}$ (see the announcement [W2]).

We now assume that the group $\Gamma$ is virtually nilpotent. We can consider, on the base $M$, the $b$-smoothing operators in the $\mathcal{B}^{\infty}$-Mishenko-Fomenko calculus, $\Psi_{b, \mathcal{B}^{\infty}}^{-\infty}\left(M, \mathcal{E}^{\infty}\right)$. The main advantage in considering this refined calculus, as in the closed case treated by Lott, is that it admits a description in terms of smoothing operators on the covering $\widetilde{M}$. To describe this correspondence we need first to recall the definition of the extended $b$-stretched product $\widetilde{M}_{\mathrm{eb}}^{2}$ of $\widetilde{M}$ (cf. [LPi1]). Let $\bigcup_{j \in J} \partial \widetilde{M}_{j}=\partial \widetilde{M}$ be the decomposition of $\partial \widetilde{M}$ into all its connected components. Then $\widetilde{M}_{\mathrm{eb}}^{2}$ is obtained by blowingup ([M]) $\widetilde{M}^{2}$ along the union of all the $\partial \widetilde{M}_{j} \times R_{\gamma}^{*}\left(\partial \widetilde{M}_{j}\right)$ for $j \in J, \gamma \in \Gamma$. The extended (small) $b$-calculus $\Psi_{\mathrm{eb}}^{*}(\widetilde{M}, \widetilde{E})$ is defined (as in $[\mathrm{M}]$ ) in terms of Schwartz kernels living on the extended $b$-stretched product. We denote by $\Psi_{\mathrm{eb}, \Gamma}^{*}(\widetilde{M}, \widetilde{E})$ the space of $\Gamma$-invariant elements. With this notation a $b$-smoothing operator $\mathcal{A} \in \Psi_{b, \mathcal{B}^{\infty}}^{-\infty}\left(M, \mathcal{E}^{\infty}\right)$ corresponds to an operator $\widetilde{A}$ belonging to $\Psi_{\mathrm{eb}, \Gamma}^{-\infty}(\widetilde{M}, \widetilde{E})$ whose Schwartz kernel $\widetilde{A}\left(z, z^{\prime}\right)$ is of rapid decay as $d\left(z, z^{\prime}\right) \rightarrow+\infty$. More details about this correspondence and the extended $b$-calculus can be found in [LPi1]. From the proof of Lemma 6.1 and (4) of Proposition 2.10 we see that, in this case, we can choose the operator $\mathcal{A}_{\mathcal{P}}^{+}$ to belong to $\Psi_{b, \mathcal{B}^{\infty}}^{-\infty}\left(M, \mathcal{E}^{\infty}\right)$. Thus the operator $\not{P}_{\mathcal{P}}^{+}=\not{D}^{+}+\mathcal{A}_{\mathcal{P}}^{+}$belongs to $\Psi_{b, \mathcal{B}^{\infty}}^{1}\left(M, \mathcal{E}^{\infty,+}, \mathcal{E}^{\infty,-}\right)$. Lemma 6.1 (2) and Theorem 12.7 of [LPi1] then give "smooth" representatives, in $K_{0}\left(\mathcal{B}^{\infty}\right)$, of the index class $\operatorname{Ind}\left(\not{D}^{+}, \mathcal{P}\right)$,

$$
\operatorname{Ind}\left(\not{P}^{+}, \mathcal{P}\right)=\left[\mathcal{L}_{\infty}(\mathcal{P})\right]-\left[\mathcal{N}_{\infty}(\mathcal{P})\right] \quad \text { in } \quad K_{0}\left(\mathcal{B}^{\infty}\right)
$$

More precisely, Theorem 12.7 of [LPi1] provides the following $\mathcal{B}^{\infty}-b$-Mishenko-Fomenko decomposition for $\not{P}_{\mathcal{P}}^{+} \equiv \not{D}^{+}+\mathcal{A}_{\mathcal{P}}^{+}$, where $x$ will denote a boundary defining function for $\partial M$.

Theorem 6.5. We can find $\varepsilon>0, \mathcal{L}_{\infty}(\mathcal{P})\left[\operatorname{resp} . \mathcal{N}_{\infty}(\mathcal{P})\right]$ a sub-B $\mathcal{B}^{\infty}$-module projective of finite rank of $x^{\varepsilon} \mathcal{H}_{b}^{\infty}\left(M, \mathcal{E}^{\infty,+}\right)\left[\operatorname{resp} . x^{\varepsilon} \mathcal{H}_{b}^{\infty}\left(M, \mathcal{E}^{\infty,-}\right)\right]$ with the following properties:

(1) $\mathcal{L}_{\infty}(\mathcal{P})$ is free and we have

$$
\not_{\mathcal{P}}^{+}\left(\mathcal{L}_{\infty}(\mathcal{P})\right) \subset \mathcal{N}_{\infty}(\mathcal{P})
$$

(2) As Frechet spaces

$$
\begin{gathered}
\mathcal{L}_{\infty}(\mathcal{P}) \oplus \mathcal{L}_{\infty}^{\perp}(\mathcal{P})=\mathcal{H}_{b}^{\infty}\left(M, \mathcal{E}^{\infty,+}\right), \\
\mathcal{N}_{\infty}(\mathcal{P}) \oplus \mathcal{D}_{\mathcal{P}}^{+}\left(\mathcal{L}_{\infty}^{\perp}(\mathcal{P})\right)=\mathcal{H}_{b}^{\infty}\left(M, \mathcal{E}^{\infty,-}\right)
\end{gathered}
$$


(3) The orthogonal projection of $\mathcal{H}_{b}^{\infty}\left(M, \mathcal{E}^{\infty,+}\right)$ onto $\mathcal{L}_{\infty}(\mathcal{P})$ and the projection $P_{\mathcal{N}_{\infty}(\mathcal{P})}$ of $\mathcal{H}_{b}^{\infty}\left(M, \mathcal{E}^{\infty,-}\right)$ onto $\mathcal{N}_{\infty}(\mathcal{P})$ along $\mathcal{D}_{\mathcal{P}}^{+}\left(\mathcal{L}_{\infty}^{\perp}(\mathcal{P})\right)$ are operators with Schwartz kernel in $\rho_{\mathrm{lb}}^{\varepsilon} \rho_{\mathrm{rb}}^{\varepsilon} \mathcal{H}_{b}^{\infty}\left(M \times M\right.$, Hom $\left(\mathcal{E}^{\infty} \otimes\right.$ $\left.{ }^{b} \Omega^{-1}, \mathcal{E}^{\infty}\right)$ ) (see $[M]$ for the definition of $\left.\rho_{\mathrm{lb}}, \rho_{\mathrm{rb}}\right)$.

(4) As Banach spaces

$$
\begin{gathered}
\mathcal{L}_{\infty}(\mathcal{P}) \otimes \Lambda \oplus \overline{\mathcal{L}_{\infty}^{\perp}(\mathcal{P}) \otimes \Lambda}=L^{2}\left(M, \mathcal{E}^{+}\right) \\
\mathcal{N}_{\infty}(\mathcal{P}) \otimes \Lambda \oplus \overline{\mathcal{P}_{\mathcal{P}}^{+}\left(\mathcal{L}_{\infty}^{\perp}(\mathcal{P})\right) \otimes \Lambda}=\mathcal{H}_{b}^{-1}\left(M, \mathcal{E}^{-}\right) .
\end{gathered}
$$

(5) The operator

$$
\not{D}_{\mathcal{P}}^{+}: \mathcal{L}_{\infty}^{\perp}(\mathcal{P}) \rightarrow \not{D}_{\mathcal{P}}^{+}\left(\mathcal{L}_{\infty}^{\perp}(\mathcal{P})\right)
$$

is invertible for the Frechet topologies; the operator

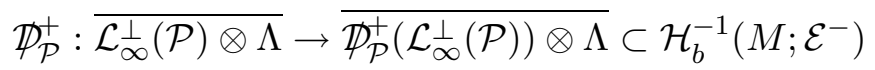

is invertible.

(6) The operator $\left(\mathcal{D}_{\mathcal{P}}^{+}\right)^{-1} \circ\left(\mathrm{Id}-P_{\mathcal{N}_{\infty}(\mathcal{P})}\right)$ belongs to the $\mathcal{B}^{\infty}$-b-calculus with bounds $\Psi_{b, \mathcal{B}^{\infty}}^{-1, \varepsilon}$.

This (precise) form of the $\mathcal{B}^{\infty}$-decomposition theorem will be used in a crucial way in the proof of the main theorem.

\section{$7 \quad b$-Chern Character, Transgression Formulae and the Higher APS Index Theorem}

We assume $\Gamma$ virtually nilpotent and adopt the notation of the previous section. Let $\mathcal{P}$ be a spectral section for the boundary operator $\mathcal{D}_{0}$ and let $\mathcal{A}_{\mathcal{P}}^{0} \in \Psi_{\mathcal{B}^{\infty}}^{-\infty}$ and $\mathcal{A}_{\mathcal{P}}^{+}(\varepsilon) \in \Psi_{b, \mathcal{B}^{\infty}}^{-\infty}$ be the perturbations constructed in Proposition 2.10 (4) and in Lemma 6.1. Define $\mathcal{A}_{\mathcal{P}}^{-}(\varepsilon)=\left(\mathcal{A}_{\mathcal{P}}^{+}\right)^{*}(\varepsilon)$ and consider the $\mathbb{Z}_{2}$-graded operator

$$
\mathcal{A}_{\mathcal{P}}(\varepsilon)=\left(\begin{array}{cc}
0 & \mathcal{A}_{\mathcal{P}}^{-}(\varepsilon) \\
\mathcal{A}_{\mathcal{P}}^{+}(\varepsilon) & 0
\end{array}\right) .
$$

The notation $\mathcal{A}_{\mathcal{P}}(\varepsilon)$ is introduced here in order to recall that this operator depends on $\varepsilon$ through the function $\widehat{\rho_{\varepsilon}}$ of Lemma 6.1. We consider the perturbed $b$-superconnection

$$
\mathbb{A}_{s, \mathcal{P}}(\varepsilon)=\Upsilon \nabla+s\left(\not \mathcal{D}+\varphi(s) \mathcal{A}_{\mathcal{P}}(\varepsilon)\right),
$$

with $\Upsilon$ equal to the grading operator. According to our discussion before Theorem 6.5 , the operator $\mathcal{A}_{\mathcal{P}}(\varepsilon) \in \Psi_{b, \mathcal{B}^{\infty}}^{-\infty}\left(M, \mathcal{E}^{\infty}\right)$ corresponds to a $\Gamma$-invariant rapidly decreasing smoothing operators $\widetilde{A}_{\mathcal{P}}(\varepsilon)$ in $\Psi_{\mathrm{e} b, \Gamma}^{-\infty}(\widetilde{M}, \widetilde{E})$ 
Hence corresponding to (7.1) there is an operator on the covering $\widetilde{\mathbb{A}}_{s, \mathcal{P}}(\varepsilon)=$ $\Upsilon \nabla+s\left(\widetilde{D}+\varphi(s) \widetilde{A}_{\mathcal{P}}(\varepsilon)\right)$.

We have proved in [LPi1] (cf. Theorem 10.6 and the remark following Proposition 12.5) that the heat kernel $\exp \left(-s^{2} \widetilde{D D}^{2}\right)$ belongs to $\Psi_{\mathrm{e} b, \Gamma}^{-\infty}(\widetilde{M}, \widetilde{E})$ with Schwartz kernel rapidly decreasing. If we view $\widetilde{\mathbb{A}}_{s, \mathcal{P}}^{2}(\varepsilon)$ as a "perturbation" of $s^{2} \widetilde{\not D}^{2}$ we can then define the heat kernel $\mathrm{e}^{-\widetilde{\mathbb{A}}_{s, \mathcal{P}}^{2}(\varepsilon)}$ using Duhamel's expansion. Proposition 13.3 of [LPi1] (the $b$-analogue of Proposition 3.4 above) can then be employed to show that

$$
\exp \left(-\mathbb{A}_{s, \mathcal{P}}^{2}(\varepsilon)\right) \in \Psi_{b, \widehat{\Omega}_{*}\left(\mathcal{B}^{\infty}\right)}^{-\infty}\left(M, \mathcal{E}^{\infty} \otimes_{\mathcal{B}^{\infty}} \widehat{\Omega}_{*}\left(\mathcal{B}^{\infty}\right)\right)
$$

It follows that we can consider its $b-\mathrm{STR}$ with value in $\overline{\widehat{\Omega}}_{*}\left(\mathcal{B}^{\infty}\right)$; we briefly recall the definition. Let us fix a trivialization $\nu \in C^{\infty}\left(\partial M ; N_{+} \partial M\right)$ of the normal bundle and $x \in C^{\infty}(M)$ a boundary defining function for $\partial M$ such that $d x \cdot \nu=1$ on $\partial M$. Recall ([Lo1]) that for any $z \in M$ and any $A_{z} \in \operatorname{End}_{\widehat{\Omega}_{*}\left(\mathcal{B}^{\infty}\right)}\left(\mathcal{E}_{z} \otimes_{\mathcal{B}^{\infty}} \widehat{\Omega}_{*}\left(\mathcal{B}^{\infty}\right)\right)$ there is a well defined supertrace $\operatorname{Str}\left(A_{z}\right) \in \overline{\widehat{\Omega}}_{*}\left(\mathcal{B}^{\infty}\right)$ (this is the even analogue of what we have explained in sect. 3$)$. We then have, by definition,

$$
\begin{gathered}
b-\operatorname{STRe} \mathrm{e}^{-\mathbb{A}_{s, \mathcal{P}}^{2}(\varepsilon)}=\int_{\Delta_{b}}^{\nu} \operatorname{Str}\left(\mathrm{e}^{-\mathbb{A}_{s, \mathcal{P}}^{2}(\varepsilon)}\right)_{\mid \Delta_{b}} \\
\equiv \lim _{\varepsilon \rightarrow 0^{+}}\left[\int_{x>\varepsilon} \operatorname{Str}\left(\mathrm{e}^{-\mathbb{A}_{s, \mathcal{P}}^{2}(\varepsilon)}\right)_{\mid \Delta_{b}}+\log \varepsilon \int_{\partial M} \operatorname{Str}\left(\mathrm{e}^{-\mathbb{A}_{s, \mathcal{P}}^{2}(\varepsilon)}\right)_{\mid \partial \Delta_{b}}\right] .
\end{gathered}
$$

Definition 7.1. The $b$-Chern character associated to $\mathbb{A}_{s, \mathcal{P}}(\varepsilon)$ is given by

$$
b-\operatorname{ch}\left(\mathbb{A}_{s, \mathcal{P}}(\varepsilon)\right)=b-\operatorname{STR}\left(\exp \left(-\mathbb{A}_{s, \mathcal{P}}^{2}(\varepsilon)\right)\right) .
$$

The next proposition and its (obvious) corollary are a key step in the proof of the higher APS index theorem. The proof of the proposition is essentially the same as the one of formula (13.21) of [MPi2]; it relies on the $b$-trace identity in the $\Gamma$-covering context as stated in Propositions 6.3 and 13.5 of [LPi1]. The easy details will be left to the reader. For simplicity we drop the $\varepsilon$ in the notation of the superconnection.

Proposition 7.2. The following transgression formula holds in $\widehat{\widehat{\Omega}}_{*}\left(\mathcal{B}^{\infty}\right)$ for any real $s>0$,

$$
\frac{d}{d s}\left(b-\operatorname{ch}\left(\mathbb{A}_{s, \mathcal{P}}\right)\right)=-d\left(b-\operatorname{STR}\left(\frac{d \mathbb{A}_{s, \mathcal{P}}}{d s} \exp \left(-\mathbb{A}_{s, \mathcal{P}}^{2}\right)\right)\right)-\frac{1}{2} \widehat{\eta}_{\mathcal{P}}(\varepsilon, s)
$$


with

$$
\begin{gathered}
\widehat{\eta}_{\mathcal{P}}(\varepsilon, s)=\frac{i}{\pi} \int_{\mathbb{R}} \operatorname{STR}_{\mathrm{Cl}(1)}\left[\partial_{\lambda}\left(I\left(\widetilde{\not D}+\mathcal{A}_{\mathcal{P}}(\varepsilon), \lambda\right)\right) \circ I\left(\frac{\left.d \mathbb{A}_{s, \mathcal{P}}, \lambda\right)}{d s}, \lambda\right)\right. \\
\left.\circ \exp \left(-I\left(\mathbb{A}_{s, \mathcal{P}}^{2}, \lambda\right)\right)\right] d \lambda .
\end{gathered}
$$

COROLlary 7.3. Let $u>t>0$; then in $\widehat{\widehat{\Omega}}_{*}\left(\mathcal{B}^{\infty}\right)$

$$
\begin{aligned}
b-\operatorname{ch}\left(\mathbb{A}_{u, \mathcal{P}}\right)=b & -\operatorname{ch}\left(\mathbb{A}_{t, \mathcal{P}}\right)-\frac{1}{2} \int_{t}^{u} \widehat{\eta}_{\mathcal{P}}(\varepsilon, s) d s \\
& -d \int_{t}^{u} b-\operatorname{STR}\left(\frac{d \mathbb{A}_{s, \mathcal{P}}}{d s} \exp \left(-\mathbb{A}_{s, \mathcal{P}}^{2}\right)\right) d s .
\end{aligned}
$$

The boundary correction term given by (7.4) is rather complicated: the next proposition shows that, up to exact forms, it is equal to the $\mathcal{P}$-higher eta invariant $\widehat{\eta}_{\mathcal{P}}$ introduced in Theorem 3.6 (2).

Proposition 7.4. (1) The following integral is absolutely convergent and well defined as an element of $\widehat{\widehat{\Omega}}_{*}\left(\mathcal{B}^{\infty}\right)$

$$
\widehat{\eta}_{\mathcal{P}}(\varepsilon)=\int_{0}^{\infty} \widehat{\eta}_{\mathcal{P}}(\varepsilon, s) d s .
$$

(2) For each $\varepsilon>0$ the difference $\widehat{\eta}_{\mathcal{P}}-\widehat{\eta}_{\mathcal{P}}(\varepsilon)$ is an exact form in $\widehat{\widehat{\Omega}}_{*}\left(\mathcal{B}^{\infty}\right)$.

Proof. (1) We will prove only the convergence on $[2,+\infty[$, the integrability on ]0,2] being much easier. We consider the following two matrixes:

$$
\gamma=\left(\begin{array}{cc}
0 & -i \\
i & 0
\end{array}\right) \quad \sigma=\left(\begin{array}{ll}
0 & 1 \\
1 & 0
\end{array}\right) .
$$

Using Lemma 6.1, we see that

$$
I\left(\mathbb{A}_{s, \mathcal{P}}, \lambda\right)=s\left[\gamma \lambda+\sigma \widetilde{D}_{0}+\sigma \varphi(s) \widehat{\rho}_{\varepsilon}(\lambda) \widetilde{A}_{P}^{0}\right]+\Upsilon \nabla .
$$

Using the two formulas $\gamma \circ \sigma+\sigma \circ \gamma=0, \Upsilon \circ \gamma+\gamma \circ \Upsilon=0$, one checks easily that

$$
-I\left(\mathbb{A}_{s, \mathcal{P}}^{2}, \lambda\right)=-s^{2} \lambda^{2}-s^{2}\left[\sigma \widetilde{D}_{0}+\sigma \varphi(s) \widehat{\rho}_{\varepsilon}(\lambda) \widetilde{A}_{P}^{0}\right]^{2} .
$$

Let us recall (cf. equation (6.1)) that $\widehat{\rho}_{\varepsilon}(0)=1$, so we can find $\delta_{1}>0$ such that $\widetilde{D}_{0}+\widehat{\rho}_{\varepsilon}(\lambda) \widetilde{A}_{P}^{0}$ is "uniformly" invertible for $\lambda \in\left[-\delta_{1}, \delta_{1}\right]$. So we can apply a version with parameters $\lambda \in\left[-\delta_{1}, \delta_{1}\right]$ of Lemma 3.7 (1) to obtain the existence of a real $\eta>0$ such that the $(s, \lambda)$-family of operators $\mathrm{e}^{-s^{2}\left(\sigma \widetilde{D}_{0}+\sigma \widehat{\rho}_{\varepsilon}(\lambda) \widetilde{A}_{P}^{0}\right)^{2}}$ satisfy the decay property (DP) with respect to $\mathrm{e}^{-s^{2} \eta}$, 
$(s, \lambda)$ being in $\left[2,+\infty\left[\times\left[-\delta_{1}, \delta_{1}\right]\right.\right.$. Now we proceed exactly as in the proof of Theorem 3.6 (1) and get the convergence of

$$
\int_{2}^{+\infty}\left[\int_{-\delta_{1}}^{\delta_{1}} \partial_{\lambda}\left(I\left(\widetilde{D}+\mathcal{A}_{\mathcal{P}}(\varepsilon), \lambda\right)\right) \circ I\left(\frac{d \mathbb{A}_{s, \mathcal{P}}}{d s}, \lambda\right) \circ \exp \left(-I\left(\mathbb{A}_{s, \mathcal{P}}^{2}, \lambda\right)\right) d \lambda\right] d s .
$$

Unfortunately, $\widetilde{D}_{0}+\widehat{\rho}_{\varepsilon}(\lambda) \widetilde{A}_{P}^{0}$ will not be "uniformly" invertible for $\lambda \epsilon$ $\mathbb{R} \backslash\left[-\delta_{1}, \delta_{1}\right]$. But the operators $\left(\sigma \widetilde{I}_{0}+\sigma \widehat{\rho}_{\varepsilon}(\lambda) \widetilde{A}_{P}^{0}\right)^{2}+\frac{\delta_{1}^{2}}{4}$ Id are certainly "uniformly" invertible for $\lambda \in \mathbb{R} \backslash\left[-\delta_{1}, \delta_{1}\right]$. So, we can adapt easily the proof of Lemma 3.7 in order to show that the operators $\mathrm{e}^{-s^{2}\left(\sigma \widetilde{D}_{0}+\sigma \widehat{\rho_{\varepsilon}}(\lambda) \widetilde{A}_{P}^{0}\right)^{2}-\delta_{1} \frac{s^{2}}{4}}$ satisfy the property (DP) uniformly with respect to $1,(s, \lambda)$ being in $\left[2,+\infty\left[\times\left(\mathbb{R} \backslash\left[-\delta_{1}, \delta_{1}\right]\right)\right.\right.$. Moreover we observe that for each integer $k$

$$
\int_{2}^{+\infty}\left[\int_{|\lambda| \geq \delta_{1}}|\lambda|^{k} \exp \left(-s^{2}\left(\lambda^{2}-\frac{\delta_{1}^{2}}{4}\right)\right) d \lambda\right] d s<+\infty .
$$

Therefore using the definition of $\widehat{\eta}_{\mathcal{P}}(s)$ given in Proposition 7.2 we get immediately the convergence of $\int_{2}^{+\infty} \widehat{\eta}_{\mathcal{P}}(s) d s$. Proposition $7.4(1)$ is thus proved.

Now we are going to prove Proposition 7.4 (2). The following variation formula associated with $\widehat{\eta}_{\mathcal{P}}(\varepsilon, s)$ may be proved exactly as in Proposition 13 of [MPi2], so we will omit its proof.

Lemma 7.5. The differential form $\widehat{\eta}_{\mathcal{P}}(\varepsilon, s)$ is smooth with respect to $(\varepsilon, s) \in$ ] $0,1] \times] 0,+\infty[$ and we have

$$
\frac{d}{d \varepsilon^{\prime}} \widehat{\eta}_{\mathcal{P}}\left(\varepsilon^{\prime}, s\right)=\frac{d}{d s}\left[\frac{1}{\pi} \int_{\mathbb{R}} \operatorname{STR}_{\mathrm{Cl}(1)}\left(s \sigma \varphi(s) \frac{d \widehat{\rho}_{\varepsilon^{\prime}}}{d \varepsilon^{\prime}} \widetilde{A}_{P}^{0} \mathrm{e}^{-I\left(\mathbb{A}_{s, \mathcal{P}}^{2}, \lambda\right)}\right) d \lambda\right]
$$

modulo exact forms and graded commutators.

Using equation (6.1), we see that $\widehat{\rho_{\varepsilon}}$ converges weakly to the Dirac delta function $\delta_{0}$ as $\varepsilon \mapsto 0^{+}$and we can use the analytic machinery developed in the proof of Theorem 3.6 (1) to check that $\int_{0}^{+\infty} \widehat{\eta}_{\mathcal{P}}(\varepsilon, s) d s$ converges to $\int_{0}^{+\infty} \hat{\eta}_{\mathcal{P}}(0, s) d s=\widehat{\eta}_{\mathcal{P}}$ in $\widehat{\widehat{\Omega}}_{*}\left(\mathcal{B}^{\infty}\right)$ as $\varepsilon \mapsto 0^{+}$. Moreover, one checks easily that the following integral:

$$
\frac{1}{\pi} \int_{\mathbb{R}} \operatorname{STR}_{\mathrm{Cl}(1)}\left[s \sigma \varphi(s) \frac{d \widehat{\rho}_{\varepsilon}}{d \varepsilon} \widetilde{A}_{P}^{0} \mathrm{e}^{-I\left(\mathbb{A}_{s, \mathcal{P}}^{2}, \lambda\right)}\right] d \lambda
$$

vanishes (as $\varphi(s)$ does!) for $s \in] 0,1]$, and converges to zero as $s \mapsto+\infty$. We get Proposition 7.4 (2) by integrating the two members of equation (7.5) with respect to $\left.\left(\varepsilon^{\prime}, s\right) \in[0, \varepsilon] \times\right] 0,+\infty[$. The proof of Proposition 7.4 is complete.

We can finally state our main result. 
Theorem 7.6. Let $\Gamma \rightarrow \widetilde{M} \rightarrow M$ be a Galois covering with boundary with the group $\Gamma$ virtually nilpotent. Let $\widetilde{D} \in \operatorname{Diff}_{b, \Gamma}^{1}(\widetilde{M}, \widetilde{E})$ be a $\Gamma$-invariant $\mathbb{Z}_{2}$-graded generalized Dirac operator as in sect. 1 . Let $\not D$ the associated $\Lambda$-differential operator. Let $\not D \in \operatorname{Diff}_{b}^{1}(M, E)$ be the Dirac operator induced on the base. Let $\widetilde{D}_{0}, \mathscr{D}_{0}, \not_{0}$ the respective boundary operators.

(1) There exists a noncommutative spectral section $\mathcal{P} \in \Psi_{\mathcal{B}^{\infty}}^{0}\left(\partial M, \mathcal{E}_{0}^{\infty}\right)$ for the boundary operator $\mathcal{D}_{0}$. The set of such noncommutative spectral sections is infinite.

(2) There is a well defined index class associated to $\not{D}$ and $\mathcal{P}, \operatorname{Ind}\left(\not{D}^{+}, \mathcal{P}\right)$, in $K_{0}\left(\mathcal{B}^{\infty}\right) \cong K_{0}\left(C_{r}^{*}(\Gamma)\right)$.

(3) For the Chern character of the index class $\operatorname{Ind}\left(\mathcal{D}^{+}, \mathcal{P}\right)$, in the noncommutative topological de Rham homology of $\mathcal{B}^{\infty}$, the following formula holds:

$$
\operatorname{Ch}\left(\operatorname{Ind}\left(\not^{+}, \mathcal{P}\right)\right)=\int_{M} \widehat{A} \wedge \operatorname{Ch}^{\prime}(E) \wedge \omega-\frac{1}{2} \widehat{\eta}_{\mathcal{P}} \text { in } \bar{H}_{*}\left(\mathcal{B}^{\infty}\right),
$$

with $\omega$ the biform introduced by Lott in [Lo1] and $\widehat{\eta}_{\mathcal{P}}$ the $\mathcal{P}$-higher eta invariant introduced in sect. 3 .

(4) If $\mathcal{Q} \in \Psi_{\mathcal{B}^{\infty}}^{0}\left(\partial M, \mathcal{E}_{0}^{\infty}\right)$ is another spectral section then

$$
\operatorname{Ch}\left(\operatorname{Ind}\left(\not^{+}, \mathcal{P}\right)\right)-\operatorname{Ch}\left(\operatorname{Ind}\left(\not^{+}, \mathcal{Q}\right)\right)=\operatorname{Ch}([\mathcal{Q}-\mathcal{P}]) \text { in } \bar{H}_{*}\left(\mathcal{B}^{\infty}\right) \text {. }
$$

Proof. The first two statements have already been discussed, see Theorem 2.7 (together with Proposition 2.8 and Corollary 2.9) and (6.5). We concentrate on (3). The proof uses the decomposition of Theorem 6.5 and proceeds as the proof of Theorem 14.1 of [LPi1]. We will omit cumbersome computations and only give the main ideas. Let $\operatorname{Ind}\left(\mathcal{D}^{+}, \mathcal{P}\right)=$ $\left[\mathcal{L}_{\infty}(\mathcal{P})\right]-\left[\mathcal{N}_{\infty}(\mathcal{P})\right] \in K_{0}\left(\mathcal{B}^{\infty}\right)$. Thus $\mathcal{F}(\mathcal{P})=\mathcal{L}_{\infty}(\mathcal{P}) \oplus \mathcal{N}_{\infty}(\mathcal{P})$ is a $\mathbb{Z}_{2^{-}}$ graded finitely generated projective $\mathcal{B}^{\infty}$-submodule of $\mathcal{H}_{b}^{1}\left(M, \mathcal{E}^{\infty}\right)$. Using $[\mathrm{K}]$ we see that $\operatorname{Ch}\left(\operatorname{Ind}\left(\not^{+}, \mathcal{P}\right)\right)=\operatorname{STR}\left(\exp \left(-\nabla_{\mathcal{F}(\mathcal{P})}^{2}\right)\right)$ with $\nabla_{\mathcal{F}(\mathcal{P})}$ equal to the compression of Lott's superconnection by the orthogonal projection onto $\mathcal{L}_{\infty}(\mathcal{P})$ and the projection onto $\mathcal{N}_{\infty}(\mathcal{P})$ along $\not_{\mathcal{P}}^{+}\left(\mathcal{L}_{\infty}(\mathcal{P})^{\perp}\right)$. We denote by $\Pi_{\mathcal{L}_{\infty}(\mathcal{P})}$ and by $P_{\mathcal{N}_{\infty}(\mathcal{P})}$ these two projections. Using part (3) of Theorem 6.5 it follows that

$$
\operatorname{STR}\left(\exp \left(-\nabla_{\mathcal{F}(\mathcal{P})}^{2}\right)\right)=b-\operatorname{STR}\left(\exp \left(-\nabla_{\mathcal{F}(\mathcal{P})}^{2}\right)\right)
$$

so that

$$
\operatorname{Ch}\left(\operatorname{Ind}\left(\not^{+}, \mathcal{P}\right)\right)=b-\operatorname{STR}\left(\exp \left(-\nabla_{\mathcal{F}(\mathcal{P})}^{2}\right)\right)
$$


Consider the $\mathcal{B}^{\infty}$-modules

$$
\begin{aligned}
& \mathcal{H}^{+}=\mathcal{H}_{b}^{\varepsilon}\left(M, \mathcal{E}^{\infty,+}\right) \oplus \mathcal{N}_{\infty}=\mathcal{L}_{\infty}^{\perp} \oplus \mathcal{L}_{\infty} \oplus \mathcal{N}_{\infty} \\
& \mathcal{H}^{-}=\mathcal{H}_{b}^{\varepsilon}\left(M, \mathcal{E}^{\infty,-}\right) \oplus \mathcal{L}_{\infty}=\mathcal{D}_{\mathcal{P}}^{+}\left(\mathcal{L}_{\infty}^{\perp}\right) \oplus \mathcal{N}_{\infty} \oplus \mathcal{L}_{\infty} \\
& \mathcal{H}=\mathcal{H}^{+} \oplus \mathcal{H}^{-} .
\end{aligned}
$$

We define the operator $\mathcal{R}_{\mathcal{P}, \alpha}^{+}: \mathcal{H}^{+} \rightarrow \mathcal{H}^{-}, \alpha \geq 0$, by

$$
\mathcal{R}_{\mathcal{P}, \alpha}^{+}(f \oplus n)=\left(\not_{\mathcal{P}}^{+} f+\alpha n\right) \oplus \alpha \Pi_{\mathcal{L}_{\infty}(\mathcal{P})} f,
$$

for each $(f \oplus n) \in \mathcal{H}^{+}$. We next define $\mathcal{R}_{\mathcal{P}, \alpha}^{-}: \mathcal{H}^{-} \rightarrow \mathcal{H}^{+}$by

$$
\mathcal{R}_{\mathcal{P}, \alpha}^{-}(g \oplus l)=\left(\not_{\mathcal{P}}^{-} g+\alpha l\right) \oplus \alpha P_{\mathcal{N}_{\infty}(\mathcal{P})} g
$$

Finally let

$$
\mathcal{R}_{\mathcal{P}, \alpha}=\left(\begin{array}{cc}
0 & \mathcal{R}_{\mathcal{P}, \alpha}^{-} \\
\mathcal{R}_{\mathcal{P}, \alpha}^{+} & 0
\end{array}\right)
$$

Denote by $\nabla^{\prime}=\nabla \oplus \nabla_{\mathcal{F}}$ the sum connection on $\mathcal{H}$ and by $\Upsilon$ the grading of $\mathcal{H}$. Define

$$
b-\operatorname{ch}_{u, \mathcal{P}, \alpha}\left(\mathcal{E}^{\infty}\right)=b-\operatorname{STR}\left(\exp \left(-\left(\Upsilon \nabla^{\prime}+u \mathcal{R}_{\mathcal{P}, \alpha}\right)^{2}\right)\right) .
$$

Clearly

$$
b-\operatorname{ch}_{u, \mathcal{P}, 0}\left(\mathcal{E}^{\infty}\right)=b-\operatorname{ch}\left(\mathbb{A}_{u, \mathcal{P}}(\varepsilon)\right)-\left[b-\operatorname{STR}\left(\exp \left(-\nabla_{\mathcal{F}(\mathcal{P})}^{2}\right)\right)\right] .
$$

Using the $b$-transgression formulae (7.3), (7.4) together with (7.9) and the short-time behaviour of the Lott superconnection heat-kernel, we then obtain the following equality in $\bar{H}_{*}\left(\mathcal{B}^{\infty}\right)$

$$
\begin{aligned}
\operatorname{Ch}\left(\operatorname{Ind}\left(\mathcal{D}^{+}, \mathcal{P}\right)\right)= & \int_{M} \widehat{A}(M) \wedge \mathrm{Ch}^{\prime}(E) \wedge \omega \\
& -\frac{1}{2} \int_{0}^{u} \widehat{\eta}_{\mathcal{P}}(\varepsilon, s) d s-\left[b-\operatorname{ch}_{u, \mathcal{P}, 0}\left(\mathcal{E}^{\infty}\right)\right] .
\end{aligned}
$$

Applying once again an obvious extension of the $b$-transgression formula we have, modulo exact forms,

$$
b-\operatorname{ch}_{u, \mathcal{P}, \alpha}\left(\mathcal{E}^{\infty}\right)=b-\operatorname{ch}_{u, \mathcal{P}, 0}\left(\mathcal{E}^{\infty}\right)+B_{1}(u, \mathcal{P}, \alpha) .
$$

with $B_{1}(u, \mathcal{P}, \alpha)$ a boundary term which can be explicitly computed. Thus, in $\bar{H}_{*}\left(\mathcal{B}^{\infty}\right)$,

$$
\begin{aligned}
\operatorname{Ch}\left(\operatorname{Ind}\left(\mathcal{D}^{+}, \mathcal{P}\right)\right)= & \int_{M} \widehat{A}(M) \wedge \mathrm{Ch}^{\prime}(E) \wedge \omega \\
& -\frac{1}{2} \int_{0}^{u} \widehat{\eta}_{\mathcal{P}}(\varepsilon, s) d s-\left(b-\operatorname{ch}_{u, \mathcal{P}, \alpha}\left(\mathcal{E}^{\infty}\right)\right)-B_{1}(u, \mathcal{P}, \alpha) .
\end{aligned}
$$


Part (5) of Theorem 6.5 shows that for $\alpha$ large enough, $\mathcal{R}_{\mathcal{P}, \alpha}^{+}$will be invertible. Thus if we replace $\nabla_{\mathcal{H}^{+}}^{\prime}$ by $\left(\mathcal{R}_{\mathcal{P}, \alpha}^{+}\right)^{-1} \circ \nabla_{\mathcal{H}^{-}}^{\prime} \circ \mathcal{R}_{\mathcal{P}, \alpha}^{+}$and leave $\nabla_{\mathcal{H}^{-}}^{\prime}$ unchanged we get a new connection denoted by $\nabla^{\prime \prime}$. Considering an homotopy of connections between $\nabla^{\prime}$ and $\nabla^{\prime \prime}$ and applying again an extension of the $b$-transgression formula we can show that

$$
\begin{aligned}
& b-\operatorname{STR}\left(\exp \left(-\left(\Upsilon \nabla^{\prime}+u \mathcal{R}_{\mathcal{P}, \alpha}\right)^{2}\right)\right) \\
& \quad=b-\operatorname{STR}\left(\exp \left(-\left(\Upsilon \nabla^{\prime \prime}+u \mathcal{R}_{\mathcal{P}, \alpha}\right)^{2}\right)\right)+B_{2}(u, \mathcal{P}, \alpha)
\end{aligned}
$$

with $B_{2}(u, \mathcal{P}, \alpha)$ an explicit boundary term. On the other hand the particular choice of $\nabla^{\prime \prime}$ shows, as in [Bi], that

$$
\begin{aligned}
& b-\operatorname{STR}\left(\exp \left(-\left(\Upsilon \nabla^{\prime \prime}+u \mathcal{R}_{\mathcal{P}, \alpha}\right)^{2}\right)\right) \\
& =b-\operatorname{TR}\left[\exp \left(-\left(\left(\Upsilon \nabla_{\mathcal{H}^{+}}^{\prime \prime}\right)^{2}+u^{2} \mathcal{R}_{\mathcal{P}, \alpha}^{-} \circ \mathcal{R}_{\mathcal{P}, \alpha}^{+}\right)\right)\right. \\
& \left.\quad-\mathcal{R}_{\mathcal{P}, \alpha}^{+} \circ \exp \left(-\left(\Upsilon \nabla_{\mathcal{H}^{-}}^{\prime}\right)^{2}-u^{2} \mathcal{R}_{\mathcal{P}, \alpha}^{+} \mathcal{R}_{\mathcal{P}, \alpha}^{-}\right) \circ\left(\mathcal{R}_{\mathcal{P}, \alpha}^{+}\right)^{-1}\right]
\end{aligned}
$$

and a last application of the $b$-transgression formula gives

$$
b-\operatorname{STR}\left(\exp \left(-\left(\Upsilon \nabla^{\prime \prime}+u \mathcal{R}_{\mathcal{P}, \alpha}\right)^{2}\right)\right)=B_{3}(u, \mathcal{P}, \alpha)
$$

with $B_{3}(u, \mathcal{P}, \alpha)$ a third explicit boundary term. Summarizing

$$
\begin{aligned}
\operatorname{Ch}\left(\operatorname{Ind}\left(\mathcal{D}^{+}, \mathcal{P}\right)\right)= & \int_{M} \widehat{A}(M) \wedge \mathrm{Ch}^{\prime}(E) \wedge \omega-\frac{1}{2} \int_{0}^{u} \widehat{\eta}_{\mathcal{P}}(\varepsilon, s) d s \\
& -B_{1}(u, \mathcal{P}, \alpha)-B_{2}(u, \mathcal{P}, \alpha)-B_{3}(u, \mathcal{P}, \alpha)
\end{aligned}
$$

in $\bar{H}_{*}\left(\mathcal{B}^{\infty}\right)$. The explicit form of the boundary terms $B_{1}, B_{2}, B_{3}$ can be found as in section 14 of [LPi1]. They all involve the heat kernel of the boundary operator $\mathbb{P}_{0}+\mathcal{A}_{\mathcal{P}}^{0}$. First it can be shown that, for algebraic reasons, $B_{1}(u, \mathcal{P}, \alpha)=0$ for each $\alpha$ and for each $u>0$. Next using the invertibility of $\mathcal{D}_{0}+\mathcal{A}_{\mathcal{P}}^{0}$ and the estimates given in the proof of Lemma 3.7 it is possible to show that for $\alpha$ large

$$
\lim _{u \rightarrow+\infty} B_{2}(u, \mathcal{P}, \alpha)=\lim _{u \rightarrow+\infty} B_{3}(u, \mathcal{P}, \alpha)=0 .
$$

Taking the limit as $u \rightarrow+\infty$ in (7.14) and recalling the convergence result stated in Proposition 7.4 we thus obtain

$\operatorname{Ch}\left(\operatorname{Ind}\left(\not^{+}, \mathcal{P}\right)\right)=\int_{M} \widehat{A}(M) \wedge \mathrm{Ch}^{\prime}(E) \wedge \omega-\frac{1}{2} \int_{0}^{+\infty} \widehat{\eta}_{\mathcal{P}}(\varepsilon, s) d s$ in $\bar{H}_{*}\left(\mathcal{B}^{\infty}\right)$.

Using Proposition 7.4 (2) and the formula above, we immediately get part (3) of Theorem 7.6. The relative index theorem, formula (7.7), follows at once from (5.1) and formula (7.6). The proof of the theorem is complete. 
Remark. We show briefly that Theorem 7.6 implies Theorem 0.1. So we assume that the $L^{2}$-spectrum of $\widetilde{D}_{0}$ has a gap at zero (see assumption (0.2) of Theorem 0.1). Let $\Pi_{0}$ be the orthogonal projection onto Ker $\not{D}_{0}$. Then, we may consider the spectral section $\mathcal{P}$ such that $\mathcal{A}_{\mathcal{P}}^{0}=\Pi_{0} ; \mathcal{P}$ is nothing but the projection onto the nonnegative part of $\mathcal{D}_{0}$ (i.e. the usual APS projection $\left.\Pi_{\geq}\right)$. We fix $x$ a boundary defining function for $\partial M$. The index class in part (3) of Theorem 0.1 is by definition $\operatorname{Ind} \not{D}^{+}(\theta)$ where $\theta$ is a fixed positive small real and $\not{D}^{+}(\theta)=x^{-\theta} \not{D}^{+} x^{\theta}$. We first show that $\operatorname{Ind}\left(\not{D}^{+}(\theta)\right)=\operatorname{Ind}\left(\not{D}^{+}, \mathcal{P}\right) \equiv \operatorname{Ind}\left(\not{D}^{+}, \mathcal{A}_{\mathcal{P}}^{+}\right)$by proving that the family $\mathscr{D}_{r}^{+}=\not{D}^{+}+r \mathcal{A}_{\mathcal{P}}^{+}+(1-r) x^{-\theta}\left[\not{D}^{+}, x^{\theta}\right]$ with $0 \leq r \leq 1$ is indeed an homotopy through Fredholm operators in the $b$-Mishenko-Fomenko calculus. Since for any $r$ the $\mathcal{D}_{r}^{+}$have the same principal symbol as that of $\not{D}^{+}$, we just have to check that the indicial family $I\left(\mathcal{D}_{r}^{+}, \lambda\right)$ remain invertible for any $\lambda \in \mathbb{R}$ and $r \in[0,1]$. Explicitly we have

$$
I\left(\mathcal{D}_{r}^{+}, \lambda\right)=\not{D}_{0}+i \lambda+r \widehat{\rho}(\lambda) \Pi_{0}+(1-r) \theta .
$$

Since $\widehat{\rho}(\lambda)$ takes real values, $I\left(\mathcal{D}_{r}^{+}, \lambda\right)$ is obviously invertible for $\lambda \neq 0$. For $\lambda=0$, we get $\not_{0}+r \Pi_{0}+(1-r) \theta$ which is clearly invertible. Thus $\operatorname{Ind}\left(\not{P}^{+}(\theta)\right)=\operatorname{Ind}(\not{D}, \mathcal{P})$. Next, with the notation of formula $(0.3)$, one can show that $\tilde{\eta}+\operatorname{Ch}\left(\mathcal{D}_{0}\right)=\widehat{\eta}_{\mathcal{P}}$ by adapting the arguments of the proof of the convergence of $\tilde{\eta}$ given in sect. 2 of [LPi1]. Summarizing, for $\theta>0$ and $\mathcal{P}$ equal to the Atiyah-Patodi-Singer spectral projection we can apply the above formulae and Theorem 7.6 and get

$$
\begin{aligned}
\operatorname{Ch}\left(\operatorname{Ind}\left(\not{D}^{+}(\theta)\right)\right) & =\operatorname{Ch}\left(\operatorname{Ind}\left(\not^{+}, \mathcal{P}\right)\right)=\int_{M} \widehat{A}(M) \wedge \operatorname{Ch}^{\prime}(E) \wedge \omega-\frac{1}{2} \widehat{\eta}_{\mathcal{P}} \\
& =\int_{M} \widehat{A}(M) \wedge \operatorname{Ch}^{\prime}(E) \wedge \omega-\frac{1}{2}\left(\tilde{\eta}+\operatorname{Ch}\left(\mathcal{D}_{0}\right)\right) .
\end{aligned}
$$

Thus Theorem 7.6 implies Theorem 0.1.

\section{References}

[APS1,2] M.F. Atiyah, V. Patodi, I. Singer, Spectral asymmetry and Riemannian geometry I, III, Math. Proc. Cambridge Philo. Soc. 77 (1975), 43-69; 79 (1976), 71-99.

[BJ] S. BAAJ, P. Julg, Théorie bivariante de Kasparov et opérateurs non bornés dans les $C^{*}$-modules Hilbertiens, C.R.Acad. Sci. Paris 296, Ser. I, (1983), 875-878.

[BaDoT] P. Baum, G. Douglas, M. Taylor, Cycles and relative cycles in analytic K-theory, J. Diff. Geom. 30 (1989), 761-804. 
[Bi] J.-M. Bismut, The Atiyah-Singer index theorem for families of dirac operators: two heat equations proofs, Inv. Math. 83 (1986), 91-151.

[BiC1,2] J.-M. Bismut, J. CheEger, Families index for manifolds with boundaries, superconnections and cones, I and II, Journ. Funct. Anal. 89 (1990), 313-363; 90 (1991), 306-354.

[BiC3] J.-M. Bismut, J. Cheeger, Remarks on the index theorem for families of Dirac operators on manifolds with boundary, Differential Geometry, (B. Lawson, K. Tenenblat, eds.), Longman Scientific, (1992), PAGENOS???

[C] J. CheEger, Spectral geometry of singular riemannian spaces, J. Diff. Geom. 18 (1983), 575-657.

[Co] A. Connes, Noncommutative Geometry, Academic Press, 1994.

[CoMo] A. Connes, H. Moscovici, Cyclic cohomology, the Novikov conjecture and hyperbolic groups, Topology 29:3 (1990), 345-388.

[DZ] X. DAI, W. Zhang, Higher spectral flow, Math. Res. Notices 3 (1996), 93-102.

[G] P. GiLkey, Invariance Theory, the Heat Equation and the Atiyah-Singer Index Theorem, Mathematical Lecture Series 11, Publish or Perish, 1984.

[Gr] M. Gromov, Groups of polynomial growth and expanding maps, Publ. Math IHES 53 (1981), 53-78.

[K] M. Karoubi, Homologie cyclique et K-théorie, Astérisque 149 (1987).

[LPi1] E. Leichtnam, P. Piazza, The $b$-pseudodifferential calculus on Galois coverings and a higher Atiyah-Patodi-Singer index theorem, Mémoires de la Société Mathématique de France 68, in press (available as Preprint ENS 96-27).

[LPi2] E. Leichtnam, P. Piazza, Higher Atiyah-Patodi-Singer index theory on Galois coverings, C.R.A.S. Série 1, t. 325 (1997), 399-404.

[Lo1] J. LотT, Superconnections and higher index theory, GAFA 2 (1992), 421-454.

[Lo2] J. LotT, Higher eta invariants, K-Theory 6 (1992), 191-233.

[Lu G. Lustzig, Novikov's higher signature and families of elliptic operators, J. Diff. Geom. 7 (1971), 229-256.

[M] R. Melrose, The Atiyah-Patodi-Singer Index Theorem, A. and K. Peters, 1993.

[MPi1] R. Melrose, P. Piazza, Analytic K-theory on manifolds with corners, Adv. in Math. 92 (1992), 1-26.

[MPi2] R. Melrose, P. Piazza, Family of Dirac operators, boundaries and the b-calculus, J. Diff. Geom. 146:1 (1997), 99-180.

[MPi3] R. Melrose, P. Piazza, An index theorem for families of Dirac operators on odd-dimensional manifolds with boundary, J. Diff. Geom. 146:2 (1997), 287-334. 
[Mif] A. Mishenko, A. Fomenko, The index of elliptic operators over $C^{*}$ algebras, Izv. Akad. Nauk SSSR, Ser. Mat. 43, (1979), 831-859.

[Q] D. Quillen, Superconnections and the Chern character, Topology 24 (1985), 89-95.

[R] J. Roe, Elliptic Operators, Topology and Asymptotic Methods, Longman Scientific and Technical, 1988. .

[T] M. TAYlor, Pseudodifferential Operators, Princeton University Press, 1981.

[W1] F. WU, The noncommutative spectral flow, http://www.ksu.edu/ fangbing, preprint 1996.

[W2] F. WU, The higher $\Gamma$-index for coverings of manifolds with boundary, http://www.ksu.edu/ fangbing, preprint 1996.

Eric Leichtnam

École Normale Supérieure

DMI, 45 rue d'Ulm

F-75230 Paris

France

E-mail: Eric.Leichtnam@ens.fr

\section{Paolo Piazza}

Università di Roma "La Sapienza"

Istituto "G. Castelnuovo"

P. le A. Moro 2

I-00185 Roma

Italy

piazza@mat.uniroma1.it

Submitted: January 1997

Final version: July 1997 